\title{
Mitochondria at the neuronal presynapse in health and disease
}

Michael J. Devine and Josef T. Kittler

Department of Neuroscience, Physiology and Pharmacology, University College London, London, WC1E 6BT, UK

michael.devine@ucl.ac.uk

j.kittler@ucl.ac.uk

\begin{abstract}
I Synapses enable neurons to communicate with each other and are therefore prerequisite to normal brain function. Presynaptically, this communication requires energy and generates large fluctuations in calcium concentrations.

Mitochondria are optimised for supplying energy and buffering calcium, and they are actively recruited to presynapses. However, not all presynapses contain mitochondria, so how might synapses with and without mitochondria differ? Mitochondria are also increasingly recognised to serve additional functions at the presynapse. Here, we discuss the importance of presynaptic mitochondria in maintaining neuronal homeostasis and how dysfunctional presynaptic mitochondria might contribute to the development of disease.
\end{abstract}


Neurons are highly specialised cells that communicate with each other by electrical and chemical means at synapses. At chemical synapses, neurotransmittercontaining synaptic vesicles are rapidly emptied into the synaptic cleft in response to an influx of $\mathrm{Ca}^{2+}$ ions through voltage-gated $\mathrm{Ca}^{2+}$ channels, triggered by action potentials ${ }^{1}$ (FIG. 1). Maintaining electrochemical gradients and releasing and recycling synaptic vesicles are all highly energy-demanding processes. In addition, these processes are all regulated by $\mathrm{Ca}^{2+}$ signalling. Thus, $\mathrm{Ca}^{2+}$ levels must be tightly controlled in the presynaptic compartment, both spatially and temporally.

Furthermore, to carry out the processing of information that is central to nervous system function, neurons must have a highly organised structure, with synapses positioned at variable locations away from the cell body, where the nucleus resides. Thus, neurons need a mechanism whereby local energy usage can be spatially matched to energy production and to buffer $\mathrm{Ca}^{2+}$.

Mitochondria are ideally suited to support this spatial variability in metabolic demand and $\mathrm{Ca}^{2+}$ buffering because they generate ATP (via oxidative phosphorylation) and take up $\mathrm{Ca}^{2+}$ into the mitochondrial matrix. Furthermore, neuronal mitochondria form a highly dynamic and adaptable network with individual organelles forming a wide range of sizes and shapes ${ }^{2}$. Indeed, live-cell imaging of mitochondria in vitro and in vivo has demonstrated that their spatial configuration, both in terms of position and morphology, continually changes over time ${ }^{3}$.

It is no surprise therefore that mitochondria are actively recruited to, and maintained at, presynapses, but such recruitment and maintenance are not ubiquitously observed, as not all neuronal presynapses are occupied by a mitochondrion ${ }^{4-9}$. Therefore, what might be the functional impact of presynaptic mitochondria? The prevailing view is that they have two canonical roles: ATP provision and $\mathrm{Ca}^{2+}$ buffering. However, this view prompts further questions: are mitochondria required at synapses under basal conditions or only during increased activity? Are there other roles for mitochondria at the presynapse? How might these be compromised in disease?

In this Review, we outline the mechanisms governing mitochondrial recruitment and their trafficking to and from presynapses and discuss their role in synaptic development and pruning. We then consider ATP provision and $\mathrm{Ca}^{2+}$ buffering by mitochondria in the presynaptic compartment and in the context of synaptic transmission, before highlighting other putative roles for these organelles, some of which depend upon their canonical functions. We also discuss how presynaptic mitochondrial dysfunction could contribute to the pathogenesis of disease, focusing on neurodegenerative conditions, as this area has attracted the most scrutiny. We conclude by highlighting future research directions, which will help illuminate some of the many unknowns in this field.

\section{Trafficking to and from the presynapse}

During eukaryotic evolution, most mitochondrial proteins came to be encoded in the nuclear genome, leaving only a handful to be encoded by mitochondrial DNA. Although mitochondria can replicate their DNA locally in axons ${ }^{10}$, mitochondrial biogenesis mainly takes place near the nucleus ${ }^{11}$. These organelles are then actively trafficked to where they are needed in the cell. This ability to transport mitochondria is particularly important in cells with complex and variable morphology such as neurons.

In cultured hippocampal neurons, $10-20 \%$ of axonal mitochondria are motile, and 
their transport occurs at velocities ranging from 0.2 to $2 \mu \mathrm{m} / \mathrm{s}^{6,12}$. In the mammalian periphery, electrical stimulation increases the proportion of mitochondria that are motile, and their velocity, with anterograde movement specifically enhanced as stimulation is ramped up ${ }^{13}$. With maturation, the proportion of motile mitochondria substantially decreases in cortical neurons in vitro ${ }^{14}$ and in the cortex and retina in vivo $^{8,14,15}$, and as mitochondria become less mobile, they spend more time at presynaptic boutons $8,14,15$. This decline in motility is paralleled by increased expression of syntaphilin (an axonal mitochondrial docking protein) with neuronal age $^{16}$.

The long-distance movement of mitochondria from the soma to presynapses (anterograde movement) and back (retrograde movement) is largely conducted via the microtubule network and a set of motor proteins (FIG. 2). Anterograde movement is mediated by kinesin motors, whereas retrograde movement is mainly mediated by dynein motors. Trafficking kinesin-binding proteins (TRAKs) are also required for mitochondrial motility and interact with kinesin heavy chain isoform 5 (KIF5) and dynein ${ }^{17,18}$. Mitochondrial motility is tightly regulated by the mitochondrial Rho GTPases (MIROs). MIRO1 and MIRO2 feature two GTPase domains that flank two EF-hand, $\mathrm{Ca}^{2+}$-sensing domains, and a C-terminal domain localising the protein to the outer mitochondrial membrane. Miro, the Drosophila melanogaster orthologue of the mammalian MIROs, is required for anterograde transport to synapses ${ }^{19,20}$, as well as dynein-driven transport into dendrites, and this movement is dependent on its $\mathrm{N}$ terminal GTPase domain ${ }^{21}$. Thus, MIRO proteins provide a potential interface by which mitochondria interact with a range of molecular motors and their regulating partners.

MIRO1 coordinates mitochondrial trafficking by sensing $\mathrm{Ca}^{2+}$, via it's EF hand domains, which enables mitochondria to stop at areas of increased [Ca $\left.{ }^{2+}\right]$. Precisely how MIRO1 achieves this is not completely understood. One possible explanation is that the increasing $\left[\mathrm{Ca}^{2+}\right]$ inhibits an interaction between MIRO1 and KIF5, releasing mitochondria from trafficking machinery (FIG. 2a) ${ }^{22}$. Alternatively, a study in mammalian cells and $D$. melanogaster showed that Miro can interact with KIF5 via Milton (the fly orthologue of TRAKs) and that increasing the $\left[\mathrm{Ca}^{2+}\right]$ released KIF5 from microtubules (FIG. 2b) ${ }^{23}$. However, recent studies in Miro1-knockout mice showed that mitochondrial movement could still be inhibited by increasing $\left[\mathrm{Ca}^{2+}\right]^{24}$ and, although axonal trafficking of mitochondria was markedly slowed in Miro1knockout hippocampal neurons, the spatial distribution of mitochondria was unchanged in axons ${ }^{25}$. These findings suggest that MIRO2 might be sufficient for mitochondrial stopping. A third possibility is that, upon binding to $\mathrm{Ca}^{2+}, \mathrm{MIRO} 1$ detaches from KIF5 which can then bind syntaphilin, anchoring mitochondria to microtubules (FIG. 2c) ${ }^{12}$. MIRO1 also enhances mitochondrial $\mathrm{Ca}^{2+}$ uptake via the mitochondrial calcium uniporter $(\mathrm{MCU})$ and the resulting increase in matrix $\left[\mathrm{Ca}^{2+}\right]$ can itself act as a signal to slow mitochondrial trafficking ${ }^{26}$.

MIROs and TRAKs can also interact with the mitofusins, which regulate mitochondrial fusion ${ }^{27}$. MIRO1 overexpression increases mitochondrial length in neurons ${ }^{28}$, an alteration which is further enhanced by overexpressing EF-hand domain mutants (suggesting that basal $\mathrm{Ca}^{2+}$ may inhibit mitochondrial fusion) and reproduced by overexpression of a constitutively active GTPase form of MIRO128,29. Thus, MIROs are capable of coordinating mitochondrial fusion-fission with trafficking.

MIRO function is prerequisite to neuronal health: Miro1 knockout in mice is lethal in the early postnatal period ${ }^{24,25}$. Moreover, conditional knockout of this gene leads to degeneration of corticospinal neurons and certain cranial nerve nuclei24; these neurons show impaired retrograde mitochondrial transport but unaffected 
mitochondrial ATP generation and $\mathrm{Ca}^{2+}$ buffering. Similarly, conditional knockout of Miro1 preferentially depletes dendritic mitochondria, compromising neuronal survival ${ }^{25}$. These reports highlight that perturbation of mitochondrial trafficking alone can lead to neuronal loss.

Glucose can also regulate mitochondrial position. TRAKs can form a complex with $O$ linked $\mathrm{N}$-acetylglucosamine (O-GlcNAc) transferase (OGT), the activity of which depends on glucose availability. In fly and rodent neurons, the $O$-GlcNAcylated state of Milton and TRAK1, respectively, is increased by extracellular glucose and, although the precise mechanism is not yet understood, this posttranslational alteration of a key mitochondrial adaptor protein is capable of leading to mitochondrial arrest ${ }^{30}$. Furthermore, surface glucose transporter levels increase in the presynaptic compartment during synaptic activity and action-potential firing, thereby increasing local glucose uptake ${ }^{31,32}$. Therefore, mitochondria can be positioned at locations of increased glucose availability, which may help optimise ATP delivery via oxidative phosphorylation.

These signals act cooperatively to position mitochondria at presynapses. Once there, mitochondria may undergo functional and structural specialisation: synaptic mitochondria are morphologically different to mitochondria elsewhere, being smaller and having increased motility 5 and a higher ratio of cristae to outer membrane surface area, which is consistent with a higher metabolic demand ${ }^{33}$. Synaptic mitochondria also have a distinct metabolic profile: they are more sensitive to complex I inhibition ${ }^{34}$ and $\mathrm{Ca}^{2+}$ overload than nonsynaptic mitochondria ${ }^{35}$, possibly owing to their relative isolation, older age and accumulated exposure to oxidative damage, which renders them susceptible to elimination (BOX 1).

\section{Synapse development and pruning}

For neuronal circuits to function properly, neuronal connections need to be established and appropriately remodelled in response to changing demands. Local protein synthesis is increasingly recognised to play a critical role in the development and remodelling of synapses ${ }^{36}$, and mitochondria are prime candidates for supplying the energy to fuel these processes. Indeed, axonal branching of chick dorsal root ganglia in response to exogenous nerve growth factor occurs at sites with stalled mitochondria, mediated by the phosphatidylinositol 3-kinase (PI3K) pathway ${ }^{37}$. Given the developmental coupling between presynaptic specialisation and axon branching and stabilisation 38,39 , it is conceivable that mitochondria could also fuel the maturation of presynapses. Indeed, branching in mouse cortical axons is mediated by liver kinase B1 (LKB1; also known as STK11) and NUAK family SNF1-like kinase 1 (NUAK1) and these kinases are necessary and sufficient to immobilise mitochondria at nascent presynaptic sites ${ }^{40}$. It is not yet established how these captured mitochondria drive axonal branching, but ATP provision seems to be the likely mechanism: mitochondria provide the ATP that is essential for assembly of the presynaptic specialisation at the Xenopus laevis neuromuscular junction and inhibition of ATP synthase here blocks clustering of synaptic vesicles and local Factin assembly that are induced by growth factors ${ }^{41}$. The anti-apoptotic factor BCL-XL has been shown to drive presynaptic maturation via increasing the GTPase activity of dynamin-related protein 1 (DRP1; also known as DLP1), a key regulator of mitochondrial fission ${ }^{42}$, thereby enhancing presynaptic mitochondrial recruitment ${ }^{43}$.

The targeted elimination of synapses is a crucial step in the maturation of neuronal circuits as the surfeit of synapses formed during development is reduced in number to those required ${ }^{44-46}$. Neuronal activity is a key driver of this process ${ }^{47-50}$ and, 
unsurprisingly, mitochondria are involved in mediating it ${ }^{51}$. Mitochondrial activation of caspase 3 plays a role in the pruning of postsynaptic spines ${ }^{52}$ and a recent study has showed that it may also have a role in the elimination of presynaptic structures.

Triggering of the apoptotic cell death pathway by axonal mitochondria promotes the selective elimination of presynaptic structures in Caenorhabditis elegans during development, via F-actin disassembly ${ }^{53}$ that is mediated by $\mathrm{Ca}^{2+}$-regulated gelsolin proteins. This pathway was discovered through an unbiased genetic screen revealing that CED3 (the worm homologue of human caspase 3) loss of function led to major defects in the localisation of presynaptic components.

\section{Provision of ATP}

Glycolysis is capable of liberating cellular energy directly from the breakdown of glucose, and certain neuronal functions exhibit a preference for this method of fuel generation. For example, the fast axonal transport of vesicles relies on glycolysis, and vesicles are furnished with their own set of glycolytic enzymes ${ }^{54}$. However, during development, neurons (from rodents at least) use ketone bodies as their primary fuel source ${ }^{55}$, which require mitochondria for their metabolism and the subsequent liberation of ATP ${ }^{56}$. Furthermore, induced pluripotent stem cells switch from glycolysis to oxidative phosphorylation as they differentiate into neurons ${ }^{57}$. Overall, mitochondria generate more than $90 \%$ of cellular ATP, powering the majority of functions within the cell, and oxidative phosphorylation is the main mechanism providing energy for neuronal activity ${ }^{58,59}$. Presynaptic processes (comprising $\mathrm{Ca}^{2+}$ clearance, transmitter uptake, and synaptic vesicle recycling and refilling) are all energy demanding ${ }^{60}$ (FIG. 3). But how can these highly dynamic demands be met?

Mitochondrial position impacts synaptic transmission. An early clue came from pharmacological inhibition of oxidative phosphorylation, which compromised synaptic neurotransmission during prolonged stimulation ${ }^{61}$. These findings were substantiated by genetic studies in $D$. melanogaster. Ablation of Miro in flies depleted presynapses of mitochondria, leading to synaptic depression after prolonged activity ${ }^{19}$. Similarly, mutations in Drp1 impaired synaptic transmission ${ }^{62}$. Notably, defects in mitochondrial localisation were seen in Drp1-knockout flies: most synapses in these flies contained far fewer mitochondria than did synapses in controls and failed to maintain neurotransmission when they were stimulated at high frequency, owing to a specific defect in mobilising reserve-pool vesicles to replenish the readily releasable pool. The deficit in neurotransmission could be partially rescued by the local application of ATP and the knockout phenotype could be recapitulated with drugs that block mitochondrial ATP production but not $\mathrm{Ca}^{2+}$ buffering. ATP is required for myosin ATPase to power vesicle mobilisation ${ }^{63}$ and is also converted to GTP to activate dynamin-mediated synaptic vesicle endocytosis ${ }^{64}$. These studies provide a mechanistic explanation for why ATP is important for replenishment of the readily releasable pool of vesicles.

Mice lacking Drp1 develop abnormally 65 and die after embryonic day 12.5. Cultured neurons from these mice have fewer neurites and exhibit abnormal synapse formation, possibly because mitochondria fail to distribute properly within developing neurons. Indeed, DRP1-dependent mitochondrial fission appears to be dispensable for non-polarised cells but is crucial for extremely polarised cells such as neurons ${ }^{66}$. Drp1 ablation in adult mouse neurons has been shown to impair synaptic transmission 67,68 . One of these studies also showed that Drp1 loss markedly increases the size of, and distance between, mitochondria within mouse 
hippocampal axons ${ }^{67}$. Under basal activity, ATP levels (determined by ATP fluorescence resonance energy transfer (FRET) sensors) were normal in the soma and synapses in these Drp1 knockout neurons. By contrast, synaptic ATP levels could not be maintained following repetitive stimulation, whereas ATP levels in the soma were unchanged. However, available ATP sensors have limited sensitivity making such comparisons challenging.

Mitochondrial motility affects prolonged activity. Syntabulin is a KIF5B adaptor protein that attaches to mitochondria to mediate anterograde movement along axons $^{69}$. A study showed that siRNA suppression of syntabulin in superior cervical ganglion neurons reduced mitochondrial distribution in axons and dendrites and caused synaptic dysfunction, comprising reduced basal activity, impaired transmission at high frequency, slower recovery after synaptic vesicle depletion and impaired short-term plasticity ${ }^{70}$. The provision of ATP could partially rescue transmission under intense stimulation; however, it could not rescue the deficit in basal transmission (the authors speculated that this deficit in basal transmission may owe to impairments in the transport of other presynaptic components). ATP was also needed for vesicle recruitment into the readily releasable pool. A different study showed that de novo ATP synthesis is required to maintain ATP levels during stimulation and, although glycolysis is required for maintaining basal ATP levels, both glycolysis and oxidative phosphorylation boost ATP during increased activity ${ }^{71}$. These studies point to a role for mitochondrial ATP in sustaining prolonged synaptic transmission.

Recent work highlights the importance of glycolysis in powering active synapses. In C. elegans, glycolytic enzymes localise to active synapses under conditions of energy stress ${ }^{72}$, and in rodent neurons, active synapses recruit glucose transporter type 4 (GLUT4) to the presynaptic plasma membrane to provide additional fuel for glycolysis ${ }^{32}$. It is worth noting that these mechanisms could also enable increased ATP provision from local mitochondria because glycolysis operates upstream of oxidative phosphorylation by supplying pyruvate.

Balancing synaptic efficiency and information transfer. Theoretical modelling suggests that maximising the probability of release paradoxically reduces the amount of information that is conveyed in the spike train ${ }^{73}$. Therefore, there may be a computational advantage in having variable synaptic transmission efficiency. Explanations for this variability have placed considerable emphasis on ion channels, synaptic vesicle kinetics and receptor concentration at synapses, but these factors cannot account for the dynamics of pulse-to-pulse variability (PPV) at single boutons that are responding to identical stimuli. Perhaps mitochondria can account for this variability? Presynaptic mitochondria maintain vesicle release and the ATP/ADP ratio when neurons are repetitively stimulated ${ }^{74}$. Increasing the proportion of motile mitochondria, by knocking out the mitochondrial anchor protein syntaphilin, elevated PPV, whereas syntaphilin overexpression reduced mitochondrial motility and lowered PPV ${ }^{74}$. The application of oligomycin, which blocks ATP synthesis, made mitochondria-containing boutons behave like those without mitochondria, whereas basal and evoked $\mathrm{Ca}^{2+}$ transients were equivalent in boutons with and without mitochondria ${ }^{74}$, suggesting that altered levels of ATP, but not of $\mathrm{Ca}^{2+}$, contribute to synaptic variability.

However, are nearby mitochondria necessary for local ATP provision? One study reported that mitochondrially derived ATP rapidly dispersed in axons, such that 
presynapses with and without mitochondria had an equivalent capacity for vesicle cycling ${ }^{75}$. By contrast, other studies have shown that ATP exhibits limited cytoplasmic diffusion ${ }^{76,77}$. The aforementioned study determined boutons solely on morphology, so it is possible that not all of the boutons were detected in this investigation. Interestingly, when the authors compared boutons with and without mitochondria in the same axon, lower levels of ATP were measured in mitochondria-free boutons ${ }^{75}$. In agreement, the ATPIADP ratio decreases more in boutons without mitochondria than in boutons with mitochondria after repeated stimulation, even when glucose is present $^{74}$. Although glycolysis or ATP diffusion from distant mitochondria may be sufficient for basal activity, a consistent picture emerges of presynaptic mitochondria being utilised to match the higher energy demands of prolonged, repetitive or intense stimulation. Mitochondrial ATP provision may also influence the efficiency of synaptic transmission ${ }^{74}$. This view is supported by an ultrastructural study of the rat hippocampus, which found a larger decrease in the number of synaptic vesicles in presynaptic boutons containing mitochondria than in those without after long-term potentiation (LTP) induced by theta burst stimulation ${ }^{9}$. However, the correlation between the presence of presynaptic mitochondria and synaptic vesicle depletion in this study was not as strong in adult rats compared with postnatal day 15 (P15) animals, possibly because, at $\mathrm{P} 15$, synapses are more reliant on mitochondria to metabolise ketone bodies. As we discuss next, mitochondrial $\mathrm{Ca}^{2+}$ handling may in fact be a more important regulator of synaptic vesicle release.

\section{Regulation of $\mathrm{Ca}^{2+}$ signalling}

$\mathrm{Ca}^{2+}$ plays a central role mediating intracellular signalling over diverse timescales and distances to fulfil many functions. Presynaptically, a very brief $\mathrm{Ca}^{2+}$ spike (generated by an action potential) triggers emptying of neurotransmitter-containing vesicles into the active zone (FIG. 4). This effect necessitates tight spatial and temporal regulation of the $\mathrm{Ca}^{2+}$ signal. The importance of presynaptic $\mathrm{Ca}^{2+}$ regulation is illustrated by post-tetanic potentiation (PTP): a minutes-long enhancement of synaptic transmission following high-frequency (tetanic) stimulation. PTP is important because it can cause changes in information processing, learning and behaviour. The sustained elevation of presynaptic $\left[\mathrm{Ca}^{2+}\right] \mathrm{i}$ could mediate PTP by increasing quanta (the number of synaptic vesicles released) per action potential. This "residual $\mathrm{Ca}^{2+}$ hypothesis" was originally formulated by Katz and Miledi nearly 50 years ago ${ }^{78}$. The search has been on since then to identify sources of $\mathrm{Ca}^{2+}$ that could prolong presynaptic $\left[\mathrm{Ca}^{2+}\right]_{\text {. }}$.

Neuromuscular junction. Potential modulators of the $\mathrm{Ca}^{2+}$ signal comprise plasma membrane extrusion pumps, cytoplasmic buffers, the endoplasmic reticulum (ER) and presynaptic mitochondria. For example, tetanic stimulation could load terminals with $\mathrm{Na}^{+}$, which reduces transmembrane $\mathrm{Na}^{+}$gradients and thus slows the efflux of $\mathrm{Ca}^{2+}$ via the $\mathrm{Na}^{+} / \mathrm{Ca}^{2+}$ exchanger (NCX) at the plasma membrane ${ }^{79}$. However, this hypothesis does not account for the sustained elevation of $\left[\mathrm{Ca}^{2+}\right] \mathrm{i}$ that is observed following tetanic stimulation. One study examined the relative contribution of the aforementioned modulators to the maintenance of post-tetanic presynaptic elevated $\left[\mathrm{Ca}^{2+}\right] \mathrm{i}$ at the crayfish neuromuscular junction (NMJ) and postulated that mitochondria have an important role ${ }^{80}$. Pharmacologically manipulating mitochondrial $\mathrm{Ca}^{2+}$ release had profound effects on PTP, whereas manipulating ER $\mathrm{Ca}^{2+}$ release had no effect. Reducing mitochondrial $\mathrm{Ca}^{2+}$ release reduced PTP whereas blocking mitochondrial $\mathrm{Ca}^{2+}$ uptake increased $\left[\mathrm{Ca}^{2+}\right]_{\mathrm{i}}$ during tetanic stimulation. The authors' interpretation 
was that mitochondrial $\mathrm{Ca}^{2+}$ sequestration during tetanic stimulation, followed by subsequent release, generated a minutes-long plateau of $\left[\mathrm{Ca}^{2+}\right]$. Synapses were driven at prolonged tetany in this study. A more physiological study of the lizard NMJ found that mitochondrial $\mathrm{Ca}^{2+}$ uptake coincided with a slowed increase in cytosolic $\left[\mathrm{Ca}^{2+}\right]^{81}$. Furthermore, uncoupling of the mitochondrial proton gradient with CCCP (carbonyl cyanide m-chlorophenyl hydrazine) triggered much greater increases in cytosolic $\left[\mathrm{Ca}^{2+}\right]$ in response to stimulation ${ }^{81}$, a pattern subsequently observed in mouse motor neuron terminals ${ }^{82}$. However, no difference in mitochondrial $\mathrm{Ca}^{2+}$ uptake was detected between $D$. melanogaster NMJs exhibiting high or low physiological activity, and such uptake did not limit cytosolic $\mathrm{Ca}^{2+}$ levels ${ }^{83}$, emphasising that the impact of mitochondria will vary depending on the type of presynapse, and the level of activity.

Calyx of Held. Curiously, the relationship between [ $\left.\mathrm{Ca}^{2+}\right] \mathrm{i}$ and neurotransmitter release may be different at the calyx of Held - a frequently studied excitatory synapse in the auditory pathway. This synapse is important for sound localisation, especially for sounds at high frequencies, when presynaptic $\mathrm{Ca}^{2+}$ transients need to be cleared rapidly, permitting subsequent action potentials to trigger discrete $\mathrm{Ca}^{2+}$ transients. Mitochondrial depolarisation slowed the removal of $\mathrm{Ca}^{2+}$ considerably in a calyx of Held preparation, but this lead to lowering of neurotransmitter release, which was reversed by increasing $\mathrm{Ca}^{2+}$ buffering ${ }^{84}$. The authors of this study suggest that the balance between competing $\mathrm{Ca}^{2+}$-dependent processes (for example, the interaction of $\mathrm{Ca}^{2+}$ with dynamin to inhibit endocytosis, and the activation of $\mathrm{RAB}$ GTPases by $\mathrm{Ca}^{2+}$-calmodulin to inhibit vesicle recycling or promote refilling of vesicles in the readily releasable pool after high-frequency stimulation) will determine the overall effect of $\mathrm{Ca}^{2+}$ on synaptic transmission. They propose that mitochondria accelerate recovery from synaptic depression after periods of moderate activity, and hence maintain transmission. Indeed, presynaptic terminals with lower $\mathrm{Ca}^{2+}$ buffering exhibited a slower recovery from synaptic depression and this did not owe to a lack of ATP, because this was continually available via the patch pipette. Furthermore, ATP synthase inhibition did not change the kinetics of the $\mathrm{Ca}^{2+}$ transient in response to stimulation ${ }^{84}$.

However, subsequent work on the calyx of Held suggested that the mitochondrial contribution to $\mathrm{Ca}^{2+}$ clearance was in fact very small ${ }^{85}$, and only apparent once $\mathrm{Na}^{+} / \mathrm{Ca}^{2+}$ exchange mechanisms were saturated $\left(\left[\mathrm{Ca}^{2+}\right]>2.5 \mu \mathrm{M}\right)$. However, it is unclear whether presynaptic mitochondrial occupancy was determined in this study, potentially underestimating the contribution of mitochondria to $\mathrm{Ca}^{2+}$ clearance. The same caveat applies to a study that found no difference in the probability of release at hippocampal neuronal synapses with and without mitochondria ${ }^{86}$ : here, the mitochondrial position was not imaged concurrently with vesicle release, and preand postsynaptically located mitochondria could not be distinguished.

Retinal bipolar neurons. One study in retinal bipolar neurons in goldfish found that mitochondrial uptake of $\mathrm{Ca}^{2+}$ was only consistently observed when $\left[\mathrm{Ca}^{2+}\right]_{\mathrm{i}}$ became very high (>800 $\mathrm{nM}$ ) or when plasma membrane $\mathrm{Ca}^{2+}$ ATPase (PMCA) was inhibited, which markedly slows $\mathrm{Ca}^{2+}$ transients ${ }^{87}$. The authors of this study therefore suggest that presynaptic mitochondria regulate $\mathrm{Ca}^{2+}$ only indirectly, at least in these neurons, by supplying ATP to power PMCA. A reduction in ATP availability (or in mitochondrial ATP production) also slowed the recovery of $\left[\mathrm{Ca}^{2+}\right]_{i}$ to baseline in this study. However, mitochondria were responsible for $\mathrm{Ca}^{2+}$ uptake when ATP was blocked by 
a nonhydrolyzable ATP analog. What could account for this discrepancy? The authors speculate that the synapses in their preparation might be unusual. Retinal bipolar neurons are tonically active, and mitochondria might be more usefully employed at terminals exhibiting a wider range of activity. Furthermore, in these cells, mitochondria are relatively distant from the active zone and hence their influence on $\mathrm{Ca}^{2+}$ dynamics would be predictably less. Finally, their experiments were conducted at room (rather than core body) temperature, which considerably underestimates the contribution of mitochondria to $\mathrm{Ca}^{2+}$ regulation, at least in mammalian neurons ${ }^{82}$.

Regulating basal activity. Mitochondria may also play a role in regulating basal transmission. Blocking the mitochondrial permeability transition pore (MPTP) in hippocampal neurons caused earlier and more rapid mitochondrial depolarisation and subsequent $\mathrm{Ca}^{2+}$ release, increased resting presynaptic [ $\left.\mathrm{Ca}^{2+}\right]$, increased synaptic transmission and attenuated LTP ${ }^{88}$. These effects were abolished by depolarising mitochondria before MPTP blockade. Stimulus-evoked $\mathrm{Ca}^{2+}$ transients (evoked using the paired-pulse protocol) were not affected by blocking MPTP, suggesting that mitochondria are more important for regulating baseline $\mathrm{Ca}^{2+}$ than for regulating transient changes in $\mathrm{Ca}^{2+}$.

In agreement, mitochondrial $\mathrm{Ca}^{2+}$ sequestration may also help reduce spontaneous release (that is, release not evoked by an action potential) by preventing large fluctuations in $\left[\mathrm{Ca}^{2+}\right]_{i}$. This phenomenon has been demonstrated in crayfish, frog and snake neuronal terminals ${ }^{61,80,89-91}$ and at the mouse $\mathrm{NMJ}^{92}$. Furthermore, insulin-like growth factor (IGF) signalling has recently been shown to increase the ratio of evoked to spontaneous transmission via mitochondria93. The IGF1 receptor tone modulates mitochondria to reduce basal cytoplasmic $\left[\mathrm{Ca}^{2+}\right]$ and spontaneous synaptic vesicle release, while enhancing presynaptic $\mathrm{Ca}^{2+}$ transients and the probability of release during spiking activity.

Role of the mitochondrial calcium uniporter. MCU activity is regarded as the principal mechanism of mitochondrial $\mathrm{Ca}^{2+}$ uptake. The $\mathrm{MCU}$ is regulated by a complex of proteins, including mitochondrial calcium uptake protein 1 (MICU1) and MICU2, which act as a gatekeeper, permitting passage of $\mathrm{Ca}^{2+}$ only at high local concentrations $(>3 \mu \mathrm{M})$ (reviewed in REF. ${ }^{94}$ ). A study of MCU blockade revealed that presynaptic mitochondria account for $40 \%$ of $\mathrm{Ca}^{2+}$ clearance at first sensory synapses in rats ${ }^{95}$, where $\mathrm{Ca}^{2+}$ uptake occurs in response to electrical stimulation at $\left[\mathrm{Ca}^{2+}\right]$ elevations as low as $200-300 \mathrm{nM}$. This report suggests that mitochondria have a bigger impact on presynaptic $\mathrm{Ca}^{2+}$ responses than PMCA. In a separate study, MCU ablation in primary neuronal cultures upregulated synaptic vesicle endocytosis, whereas MCU overexpression had the opposite effect; activity-dependent increases in presynaptic $\mathrm{Ca}^{2+}$ levels were unchanged by MCU knockdown ${ }^{96}$. However, presynaptic mitochondrial occupancy was not determined in this study. Thus, any mitochondrial contribution to $\mathrm{Ca}^{2+}$ buffering would be underestimated, given that many presynapses are devoid of mitochondria.

Recently, we and others reported that presynaptic mitochondria actively attenuate presynaptic $\mathrm{Ca}^{2+}$ transients in response to trains of action potentials, thereby downregulating synaptic vesicle release in hippocampal ${ }^{97}$ and cortical neurons ${ }^{98}$. These effects were ablated by blocking MCU. One of the studies identified LKB1 as a regulator of MCU abundance, and showed that MCU expression could rescue synaptic transmission defects in $L k b 1$-null neurons (comprising reduced presynaptic 
$\mathrm{Ca}^{2+}$ buffering, increased asynchronous release, and impaired short-term plasticity) ${ }^{98}$. We showed that mitochondria are differentially recruited to the presynapse during homeostatic plasticity, with enhanced recruitment when network activity is upregulated and reduced recruitment when network activity is inhibited ${ }^{97}$. This recruitment is dependent on the $\mathrm{Ca}^{2+}$-sensing EF hand domains of MIRO1, and on the buffering of $\mathrm{Ca}^{2+}$ by $\mathrm{MCU}$. This finding is consistent with previous work demonstrating that $\mathrm{MCU}$-mediated $\mathrm{Ca}^{2+}$ uptake is required for LTP in a mouse model of hyperalgesia ${ }^{99}$. Thus, mitochondrial positioning can contribute to synaptic rescaling over long timescales. We observed a threshold effect whereby mitochondria influenced $\mathrm{Ca}^{2+}$ transients only in response to trains of 20 or more action potentials ${ }^{97}$. This phenomenon might be associated with complete depletion of the readily releasable pool of synaptic vesicles (which are thought to be fully released within 40 action potentials ${ }^{100}$ ) and might relate to a requirement for a high local $\left[\mathrm{Ca}^{2+}\right]$ to trigger $\mathrm{Ca}^{2+}$ uptake via $\mathrm{MCU}$.

An active regulator of synaptic transmission. Clearly mitochondria are important players in presynaptic $\mathrm{Ca}^{2+}$ regulation. These studies highlight the importance of taking neuronal type, preparation temperature and mitochondrial position (relative to the presynapse) into account when determining mitochondrial impact on presynaptic function. Far from a passive $\mathrm{Ca}^{2+}$ sink operating remote from the presynapse, they are actively recruited to this compartment to tune synaptic activity over time. Thus, the picture becomes complex: although mitochondria are utilised to support increased activity in synapses, they can also downregulate synaptic function over a longer time scale.

How does mitochondrial $\mathrm{Ca}^{2+}$ regulation fit with provision of ATP? The two might be linked, as an increase in the former has been shown to stimulate production of the latter in mouse neuronal culture ${ }^{101}$ and cardiac myocytes ${ }^{102}$. It is not understood how this happens, but may involve increases in [Ca $\left.{ }^{2+}\right]$ mediating the activation of citric acid cycle and oxidative phosphorylation enzymes ${ }^{103}$. Overall, presynaptic mitochondria are recruited to sites of $\mathrm{Ca}^{2+}$ flux and are utilised to support prolonged activity via ATP provision, but, simultaneously, they place an upper limit on synaptic activity levels via $\mathrm{Ca}^{2+}$ uptake. This provides a powerful homeostatic mechanism: prolonged synaptic activity is linked to appropriate provision of energy substrates; however, excess activity is tempered by the buffering of $\mathrm{Ca}^{2+}$, and the processes are managed by the same organelle. Why therefore might some synapses be devoid of mitochondria? Mitochondrial motility increases synaptic variability by altering ATP homeostasis ${ }^{74}$, and recruitment of mitochondria to and from presynaptic boutons allows neurons to rescale synapses in response to long-term changes in network activity ${ }^{97}$. Thus, not being dependent upon locally positioned mitochondria could furnish neurons with greater computational flexibility.

\section{Mitochondria and the ER}

Mitochondria are associated with the ER at contact regions called mitochondriaassociated ER membranes (MAMs), which comprise 5 to $20 \%$ of the mitochondrial surface. Just as mitochondria form a network which is distributed throughout the neuron, so too does $E R$, and it is increasingly recognised that mitochondria-ER interactions can modulate a wide range of physiological processes, including ATP synthesis, autophagy, mitochondrial trafficking and apoptosis (reviewed in REF. ${ }^{104}$ ). 
help shape $\mathrm{Ca}^{2+}$ signalling via interacting with mitochondria. Recent electron microscopy in mouse brain tissue has revealed that ER forms a web surrounding mitochondria and other organelles at presynapses ${ }^{105}$. Movement of $\mathrm{Ca}^{2+}$ from ER to mitochondria can prolong the residual $\left[\mathrm{Ca}^{2+}\right]$ and promote synaptic vesicle release ${ }^{106}$. Moreover, microdomains of high $\left[\mathrm{Ca}^{2+}\right]$ exist at the mitochondria-ER interface ${ }^{107}$, which can facilitate $\mathrm{Ca}^{2+}$ uptake via $\mathrm{MCU}$.

Interestingly, Miro has recently been shown to modulate mitochondrial $\mathrm{Ca}^{2+}$ uptake, independent of its trafficking role. In flies, Miro interacts with $\mathrm{Ca}^{2+}$ transporters (for example, Porin, equivalent to mammalian voltage-dependent anion-selective channel (VDAC)) at mitochondria-ER contact sites; Miro inactivation caused mitochondrial $\mathrm{Ca}^{2+}$ depletion and metabolic impairment whereas Miro overexpression triggered mitochondrial $\mathrm{Ca}^{2+}$ overload and apoptosis ${ }^{108}$. MIRO1 also localises to mitochondrial-ER contact sites in COS7 cells, suggesting that it plays similar roles in mammalian cells ${ }^{109}$.

Recently, presynaptic ER has been shown to modulate $\mathrm{Ca}^{2+}$ in two ways: buffering of $\mathrm{Ca}^{2+}$ during repetitive firing (analogous to mitochondria), and reducing $\mathrm{Ca}^{2+}$ influx in response to single action potentials ${ }^{110}$. The latter was shown to be mediated by the $\mathrm{ER} \mathrm{Ca}{ }^{2+}$ sensor stromal interaction molecule 1 (STIM1) in response to depletion of ER $\mathrm{Ca}^{2+}\left(\mathrm{REF} .{ }^{110}\right)$. It will be important to establish how this ER-STIM1 feedback loop, and more generally MAMs, interact with mitochondria to regulate presynaptic function.

\section{Mitochondria as a signalling hub}

Mitochondrial retrograde signalling can be defined as the cell's response to changes in the functional status of mitochondria ${ }^{111}$. For example, mitochondrial dysfunction in yeast triggers the nuclear transcription of genes that supply mitochondria with substrates to help compensate for a failing citric acid cycle. In mammalian cells, altered $\mathrm{Ca}^{2+}$ signalling has been implicated as an additional mechanism by which mitochondria can report back to the cell. A specific neuronal impact has been reported in $D$. melanogaster. inducing mitochondrial damage depleted presynapses of mitochondria and reduced neuronal viability ${ }^{112}$. This effect could be rescued, without salvaging mitochondria, by blocking retrograde signalling mediated by Sima (the fly homologue of hypoxia-inducible factor 1a (HIF1a)).

Mitochondrial reactive oxygen species (ROS) exert cellular damage, but can also regulate the strength of postsynaptic $\mathrm{GABA}_{\mathrm{A}}$ receptors at inhibitory synapses of stellate cells ${ }^{113}$. Thus, cellular metabolism can be coupled to the strength of synaptic transmission. ROS can impinge on signalling pathways by oxidising key moieties on proteins to alter their activity or function. Mitochondrial ROS are now recognised to be important in hypoxia adaptation, autophagy, immunity, differentiation and longevity (reviewed in REF. ${ }^{114}$ ).

Mitochondria can also integrate and respond to other signals that can impact presynaptic function, including IGF1 signalling as outlined above ${ }^{93}$. Brain-derived neurotrophic factor has also been shown to increase mitochondrial docking at presynaptic sites, in a MIRO1-dependent manner, by increasing intracellular $\mathrm{Ca}^{2+}$ levels via PI3K and phospholipase-Cy pathways ${ }^{115}$. Intriguingly, neuronal mitochondrial membranes have been shown to contain type-1 cannabinoid receptor $\mathrm{CB}_{1}$ (a G protein-coupled receptor). Activation of these receptors leads to inhibition of presynaptic mitochondrial respiration. This contributes to regulation of hippocampal short-term plasticity ${ }^{116}$, and their knockout prevents memory impairment induced by 
cannabinoids ${ }^{117}$, providing a further link between presynaptic mitochondrial bioenergetics and neuronal function.

\section{Biosynthesis of macromolecules}

Mitochondria play a critical role in the biosynthesis of various important molecules, including lipids, hormones and neurotransmitter intermediates ${ }^{118,119}$. This biosynthetic role is implicated in $D$. melanogaster NMJ development ${ }^{120}$ : flies expressing a mutant form of the transmembrane GTPase Marf (required for correct mitochondrial fusion) had fewer presynaptic mitochondria and exhibited morphologically abnormal NMJs. The mutant NMJs also failed to maintain synaptic transmission during repetitive stimulation (although basal transmission is unaffected). The production of steroid hormones in ring glands (a major insect endocrine organ) was affected in the mutant flies and thought to be the cause of the NMJ pathology (ablating Marf solely in neurons did not lead to NMJ pathology, whereas loss of Marf in ring glands did). The phenotype was fully reversed by expressing human mitofusin-1 (MFN1) and MFN2, pointing to a conserved mechanism. MFN2 in particular has been implicated in human steroid hormone synthesis, suggesting that disordered mitochondrial hormone production might impact on mammalian synapses as well. Of note, aromatase (which synthesises oestradiol and is located in the ER) is expressed in synapses, raising the possibility of synaptocrine signalling (reviewed in REF. ${ }^{121}$ ). Indeed, mutations in optic atrophy protein 1 (OPA1) (which cause optic atrophy) have been shown to dysregulate mitochondrial steroid production in the retina, promoting apoptosis of retinal ganglion cells ${ }^{122}$.

Mitochondria are also crucial contributors to the synthesis of haem - the key component of haemoglobin and cytochromes, which regulate many signalling processes within the brain. Haem deficiency leads to neuronal senescence ${ }^{123}$ and neurite degeneration that is mediated by NMDA receptor-dependent ERK1/2 activation ${ }^{124}$, highlighting an important synaptic role for this molecule.

Frataxin is a mitochondrial protein involved in iron-sulphur complex biosynthesis, dysfunction of which leads to defective mitochondrial transport to synapses, and reduced mitochondrial membrane potential in $D$. melanogaster ${ }^{125}$. By late third instar, NMJs of affected flies accumulate excess depolarised mitochondria.

\section{Role in disease pathogenesis}

We have discussed the importance of presynaptic mitochondria to synaptic, and indeed neuronal, homeostasis. How might their dysfunction contribute to disease? Genetic links between mitochondria and disease are well established: mutations in many genes that encode proteins critical to mitochondrial function give rise to multisystem hereditary diseases known collectively as the mitochondrial cytopathies. Furthermore, impaired mitochondrial fusion resulting from mutations in OPA1 causes optic atrophy ${ }^{122}$, and from mutations in MFN2 can cause Charcot-Marie-Tooth disease type $2 \mathrm{~A}$ (a familial neuropathy) ${ }^{120}$. Defective haem biosynthesis can lead to porphyria, deleterious to neuronal function ${ }^{126}$. Mutations in $F X N$ (which encodes frataxin) lead to Friedreich's ataxia, with involvement of sensory neurons in the spinal cord $^{125}$.

But what of presynaptic mitochondria specifically? Recent work showed that mitochondrial uncoupling protein 2 (UCP2) is required for correct synaptic remodelling in response to hypoxia, which is considered important in perinatal hypoxia, epilepsy and stroke ${ }^{127}$. Furthermore, neurons are postmitotic and, apart from a few exceptions, are not replenished in adulthood ${ }^{128}$, and they are therefore 
vulnerable to premature attrition in neurodegenerative diseases, including Alzheimer disease (AD), Parkinson disease (PD) and amyotrophic lateral sclerosis (ALS) (FIG. 5). Synaptic degeneration may precede neurodegeneration. For example, a mouse model of hereditary spastic paraplegia (featuring degeneration of corticospinal axons) owing to recessive mutations in the SPG7 gene (encoding the mitochondrial ATPase paraplegin) exhibited morphological abnormalities in synaptic terminals and distal axons, which preceded degeneration but coincided with motor impairment ${ }^{129}$. Synapses might also degenerate early in the course of $A D^{130}$ and $P D^{131}$. Affected neurons may then die back, akin to Wallerian degeneration. Here, we discuss and speculate on the available evidence that presynaptic mitochondria might play a key pathogenic role in these conditions.

Alzheimer disease. AD is the most common cause of dementia and features the degeneration of cortical neurons and certain subcortical structures. The pathology of the disease includes the accumulation of extracellular plaques of the peptide amyloid- $\beta(A \beta)$ and the formation of intracellular tangles of tau ${ }^{132}$, although precisely why neurons are lost in this disease is not fully understood.

Studies in rodent cortical synaptosomes have showed that mitochondrial dysfunction can be triggered by exposure to $A \beta^{133-135}$. Furthermore, acute $A \beta$ exposure impaired mitochondrial transport in hippocampal axons ${ }^{136,137}$. In contrast, chronic exposure to mild mitochondrial stress has been shown to increase retrograde mitochondrial transport by releasing syntaphilin anchor proteins, prior to activating parkin-mediated mitophagy ${ }^{138}$. Moreover, syntaphilin release has been seen in neurons from mouse models of $A D$ and $A L S$ and in brain tissue from individuals with $A D^{138}$. Separate work has identified an increase in retrograde transport of damaged mitochondria to the soma in neurons from mouse models of $A D$ and in $A D$ human brain tissue ${ }^{139}$.

Once mitochondria are presynaptically located, AD pathology may alter the fissionfusion of mitochondria, with deleterious consequences. Nitric oxide, produced in response to exposure to $A \beta$ oligomers, triggered mitochondrial fission, synaptic loss and neurodegeneration in rodent cortical neurons, partly via S-nitrosylation of DRP1, and this modification of DRP1 was also detected at higher levels in brain tissue from individuals with $A D$ than in that from controls ${ }^{140}$. DRP1 can also interact with $A \beta$ directly ${ }^{141}$ and depletion of DRP1 reduces mitochondrial dysfunction and improves synaptic activity in a mouse model of $A D^{142}$.

Interestingly, disease-associated mutant tau appears to inhibit DRP1 activity by preventing its interaction with mitochondria (via actin stabilisation), leading to impaired fission, elongated and dysfunctional mitochondria, and inappropriate cell cycle reentry, leading to cell death ${ }^{143}$. Lowering DRP1 can improve mitochondrial and synaptic function in a mouse model of tauopathy ${ }^{144}$. Furthermore, phosphorylated tau disrupted mitochondrial transport in mouse cortical neurons ${ }^{145}$, and in $D$.

melanogaster, RNAi knockdown of milton or Miro exacerbated human tau-induced neurodegeneration and tau phosphorylation at the AD-related site Ser262 ${ }^{146}$, highlighting the synergy between defective trafficking machinery and phosphorylated tau. Tau depletion can ameliorate deficits in neuronal activity and behaviour in transgenic mouse models of $A D^{147,148}$ and it can do this by preventing $A \beta$-induced defects in axonal transport ${ }^{149}$, emphasising that axonal transport is critically important for maintaining neuronal viability.

$A \beta$ is transported into mitochondria via the translocase of the outer membrane (TOM) complex and associates with the mitochondrial inner membrane ${ }^{150}$. Cyclophilin $D$ (CypD), a key component of MPTP, has been shown to interact with mitochondrial 
$A \beta$ and to potentiate synaptic dysfunction, whereas cortical neurons deficient in CypD were more resistant to $A \beta$-induced mitochondrial dysfunction and MPTP opening, and CypD depletion alleviated synaptic dysfunction and learning and memory deficits in an AD mouse model ${ }^{151}$. A study in $D$. melanogaster has provided support for these key findings: overexpression of human $A \beta$ lead to intracellular accumulation of $A \beta$, slowed axonal mitochondrial transport, depleted presynaptic mitochondria and synaptic vesicles, and depressed synaptic transmission ${ }^{152}$. The overexpression of the 'Arctic' form of $A \beta$, a mutation found in a Swedish family with $A D$, accelerated the manifestation of these effects ${ }^{152}$.

Altered $\mathrm{Ca}^{2+}$ handling by presynaptic mitochondria at the mossy fibre synapse has been observed as an early deficit in $A D$ mouse models overexpressing a mutant form of human amyloid precursor protein $(A P P)$, from which $A \beta$ is derived, or featuring knockout of presenilin-1, which is required for normal processing of APP into $A \beta^{153,154}$. Increased IGF1 signalling has been found in brain tissue from people with $A D^{155}$ and in the hippocampi of transgenic mice overexpressing both mutant APP and PS $1^{156}$. In an AD mouse model, blockade of IGF1 signalling corrected early presynaptic dysfunction (comprising increased basal transmission but reduced shortterm plasticity, and preceding synaptic loss and $A \beta$ plaques) mediated by altered mitochondrial $\mathrm{Ca}^{2+}$ handling and ATP generation ${ }^{93}$.

AD pathology can also alter how mitochondria and the ER interact at MAMs. Upregulated MAM-associated proteins have been observed in brain tissue from individuals with $A D$ and the APP swe/Lon $_{\text {mouse model (which harbours disease- }}$ causing mutations in APP), and this was an early pathological event because it preceded the development of amyloid plaques ${ }^{157}$. Nanomolar concentrations of oligomeric $A \beta$ elevated expression of components of a mitochondrial-ER bridging complex (namely VDAC and the inositol trisphosphate receptor), increased the number of mitochondrial-ER contact points, and augmented ER-to-mitochondrial $\mathrm{Ca}^{2+}$ transfer and thus increased mitochondrial $\left[\mathrm{Ca}^{2+}\right]$ in neuroblastoma cells.

Furthermore, siRNA-mediated knockdown of one or the other of two putative MAMassociated proteins (phosphofurin acidic cluster sorting protein 2 or the sigma-1 receptor) triggered degeneration of mouse hippocampal neurons, highlighting the importance of MAM for neuronal survival.

Parkinson disease. PD is the most common neurodegenerative movement disorder, featuring loss of midbrain dopaminergic neurons, causing the clinical triad of tremor, rigidity and bradykinesia (slowness of movement). Mitochondria were first implicated in PD when complex I deficiency was observed in PD brain tissue ${ }^{158}$. The identification of mutations in PINK $1^{159}$ and PARK2 (which encodes parkin) ${ }^{160}$ as causative of autosomal recessive forms of familial PD underlined the importance of mitochondria in maintaining midbrain dopaminergic neurons. The PINK1-parkin pathway is central to mitophagy (BOX 1). In most cells, this occurs close to the soma where lysosomes are abundant. In neurons, this can be achieved by increasing movement of damaged mitochondria back towards the soma ${ }^{161}$ where parkintargeted mitochondria accumulate ${ }^{162}$. In vivo studies in $D$. melanogaster support the view that neuronal mitophagy resides largely in the soma ${ }^{163,164}$. Indeed, anterograde axonal transport of mitochondria is reduced in mouse dopaminergic neurons deficient in respiratory chain enzymes ${ }^{165}$ or exposed to the neurotoxin 1-methyl-4phenylpyridinium (MPP+), which also increases retrograde transport ${ }^{166}$. In vivo imaging in zebrafish has also revealed substantially increased retrograde movement of mitochondria in dopaminergic neurons exposed to MPP $+{ }^{167}$. These findings highlight the importance of appropriate trafficking of mitochondria in response to 
neuronal stress.

However, damaged mitochondria can also cease movement via PINK1-parkinmediated degradation of MIRO1 ${ }^{168}$. MIRO1 turnover on damaged mitochondria is altered in fibroblasts from individuals with PD bearing PARK2 mutations ${ }^{169}$. Recently, leucine-rich repeat kinase 2 (LRRK2), another PD-related protein, has been shown to bind MIRO1, aiding its degradation, whereas pathogenic mutations in LRRK2 impair such binding, delaying the arrest and subsequent removal of damaged mitochondria ${ }^{170}$. Therefore, specific disruption of mitochondrial transportation (via MIRO1-induced mitochondrial arrest) may be pathogenic in PD. Such stationary mitochondria have been shown to be capable of locally recruiting parkin, autophagosomes and lysosomes to achieve mitophagy in situ ${ }^{171}$. If this pathway is faulty, damaged mitochondria could accumulate in axons and presynapses in PD. It will be important to determine whether such local mitophagy is capable of clearing damaged mitochondria at presynapses and how this relates to disease.

Impairments in dopamine release and synaptic plasticity have also been observed in Pink1 knockout mice ${ }^{172}$. The cause of these impairments may be related to impaired mitochondrial energy production, as PINK1 deficiency compromises complex I function and, in PINK1 deficient flies, reserve pool vesicles fail to mobilise during rapid stimulation, which can be corrected with exogenous ATP ${ }^{173}$.

SNCA (encoding a-synuclein) was the first gene to be linked to familial PD ${ }^{174}$, and asynuclein is the principal protein component of Lewy bodies - a pathological hallmark of the disorder ${ }^{175}$. a-Synuclein is important in PD pathogenesis (whether sporadic or familial) but precisely how remains poorly understood. Indirect clues suggest that a-synuclein may induce presynaptic mitochondria dysfunction. aSynuclein is predominantly cytosolic, but it mislocalises to mitochondria during cellular stress ${ }^{176}$. In a hypothalamic neuronal tumour cell line, a-synuclein overexpression induces complex I inhibition and mitochondrial membrane depolarisation ${ }^{177}$. Transgenic mice expressing mutant a-synuclein show neurodegeneration with altered mitochondrial morphology and mitochondrial DNA damage ${ }^{178}$ and trafficking ${ }^{179}$. In parallel, a-synuclein accumulates presynaptically in neurodegeneration ${ }^{180}$. Furthermore, transgenic a-synuclein overexpression impairs neurotransmitter release ${ }^{181}$ and enlarges synaptic vesicles - a phenotype also observed in animal models lacking key synaptic proteins ${ }^{182}$.

The intracellular localisation of a-synuclein has been refined to reveal its presence in $\mathrm{MAM}^{183}$. Mutant a-synuclein alters this localisation and reduces mitochondrial-ER apposition, decreases MAM function, and increases mitochondrial fragmentation ${ }^{183}$. Recently, a-synuclein has been shown to bind to vesicle-associated membraneassociated protein $B(\mathrm{VAPB})^{184}$. VAPB is an ER protein whose amino-terminus projects into the cytosol and interacts with an outer mitochondrial membrane protein, namely protein tyrosine phosphatase-interacting protein 51 (PTPIP51) ${ }^{185}$. Thus, VAPB is a MAM protein and can alter how mitochondria take up $\mathrm{Ca}^{2+}$ when it is released from ER stores. Mutant a-synuclein disrupts VAPB-PTPIP51 tethering, thereby loosening mitochondria-ER interactions ${ }^{184}$. Recently, the absence of Park2 has been shown to increase ER and mitochondrial apposition, and to increase ER-tomitochondria $\mathrm{Ca}^{2+}$ transfer in mice, patient fibroblasts and patient-derived neurons ${ }^{186}$, and overabundance of MFN2 was implicated in mediating these effects.

A role for HIF1a has also been implicated in PD. Knockdown of Sima (the fly homologue of HIF1a) restored function in a $D$. melanogaster model of parkin PD ${ }^{112}$, suggesting that HIF1a signalling may be an interesting therapeutic target to explore. 
Amyotrophic lateral sclerosis. ALS is a neurodegenerative disorder that specifically affects upper and lower motor neurons. Mutations in $\mathrm{Cu} / \mathrm{Zn}$ superoxide dismutase-1 (SOD1) cause a familial form of ALS ${ }^{187}$. The pathogenic role of SOD1 mutations is not understood; however, motor neurons from mice expressing human mutant SOD1 exhibit impairment of (especially anterograde) mitochondrial axonal transport ${ }^{188}$. In this study, the mitochondria exhibited a rounder morphology and reduced membrane potential - both indicators of damage. Recently, mutant SOD1 has been reported to induce parkin-dependent degradation of MIRO1, which might explain the mitochondrial trafficking defect ${ }^{189}$. This finding also corroborates findings in Miro1 knockout mice, which feature upper motor neuron degeneration ${ }^{24}$.

A wide range of $D$. melanogaster models of genetic ALS, owing to mutations in TARDBP (which encodes TAR DNA-binding protein 43 (TDP43)), FUS or C9orf72, exhibit impaired axonal transport ${ }^{190}$, as do rodent neurons expressing ALS-causing mutant variants of VAPB ${ }^{191}$. These mutations in VAPB alter the encoded protein's ability to bind to PTPIP51 and increase mitochondrial $\mathrm{Ca}^{2+}$ uptake. Expression of wildtype TDP43, or mutant forms of TDP43 associated with familial forms of ALS, have been shown to perturb mitochondrial-ER interactions by disrupting the relationship between VAPB and PTPIP51 ${ }^{192}$. Accumulation of hyperphosphorylated, ubiquitinated and cleaved TDP-43 is a feature common to ALS and also a subset of frontotemporal dementia (FTD) ${ }^{193}$, and mutations in TARDBP cause some sporadic and familial cases of ALS ${ }^{194}$.

Whether or not the pathological processes described here in ALS also occur at the presynapse remains to be determined, but we predict that this will be a core feature of degenerating neurons, given the emerging importance of mitochondrial-ER interactions to presynaptic homeostasis outlined above. Box 2 highlights therapeutic strategies that could be explored experimentally to enhance neuronal survival via boosting presynaptic mitochondrial function.

\section{Conclusions}

Of all the many factors governing synaptic, neuronal and network activity, mitochondrial location and function have a major impact. However, if presynaptic mitochondria are so important, why are some presynapses devoid of them? Their absence may provide neurons greater computational flexibility, but this reason is not the full story. The role of mitochondria in signalling and biosynthesis as well as their interactions with the ER suggest that other factors must influence presynaptic mitochondrial occupancy. Furthermore, the regulation of trafficking of mitochondria to and from presynapses, and the full extent of mitochondrial function once there, remain incompletely understood. Consequently, their contribution to disease pathogenesis is not yet fully defined.

The impact of mitochondria on the regulation of presynaptic function highlights the need to target these organelles more specifically. The use of genetically encoded reporters of mitochondrial position, $\left[\mathrm{Ca}^{2+}\right]$ and synaptic vesicle release enables interactions between mitochondria and presynaptic function to be interrogated in real time and in vivo. This approach, combined with use of transgenic and induced pluripotent stem cell models of disease, should help us to understand how mutations that cause neurological disease can disrupt mitochondrial, synaptic and, consequently, neuronal homeostasis. 
1. Jahn, R. \& Fasshauer, D. Molecular machines governing exocytosis of synaptic vesicles. Nature 490, 201-207 (2012).

2. MacAskill, A. F. \& Kittler, J. T. Control of mitochondrial transport and localization in neurons. Trends Cell Biol. 20, 102-112 (2010).

3. Sheng, Z.-H. The Interplay of Axonal Energy Homeostasis and Mitochondrial Trafficking and Anchoring. Trends Cell Biol. 27, 403-416 (2017).

4. Shepherd, G. M. \& Harris, K. M. Three-dimensional structure and composition of CA3-->CA1 axons in rat hippocampal slices: implications for presynaptic connectivity and compartmentalization. J Neurosci 18, 83008310 (1998).

5. Chang, D. T. W., Honick, A. S. \& Reynolds, I. J. Mitochondrial trafficking to synapses in cultured primary cortical neurons. Journal of Neuroscience 26, 7035-7045 (2006).

6. Kang, J.-S. et al. Docking of axonal mitochondria by syntaphilin controls their mobility and affects short-term facilitation. Cell 132, 137-148 (2008).

7. Obashi, K. \& Okabe, S. Regulation of mitochondrial dynamics and distribution by synapse position and neuronal activity in the axon. Eur. J. Neurosci. 38, 2350-2363 (2013).

8. Smit-Rigter, L. et al. Mitochondrial Dynamics in Visual Cortex Are Limited In Vivo and Not Affected by Axonal Structural Plasticity. Current Biology 26, 2609-2616 (2016).

9. Smith, H. L. et al. Mitochondrial support of persistent presynaptic vesicle mobilization with age-dependent synaptic growth after LTP. Elife 5, e15275 (2016).

10. Amiri, M. \& Hollenbeck, P. J. Mitochondrial biogenesis in the axons of vertebrate peripheral neurons. Dev Neurobio/ 68, 1348-1361 (2008).

11. Davis, A. F. \& Clayton, D. A. In situ localization of mitochondrial DNA replication in intact mammalian cells. The Journal of Cell Biology 135, 883893 (1996).

12. Chen, Y. \& Sheng, Z.-H. Kinesin-1-syntaphilin coupling mediates activitydependent regulation of axonal mitochondrial transport. The Journal of Cell Biology 202, 351-364 (2013).

13. Sajic, M. et al. Impulse conduction increases mitochondrial transport in adult mammalian peripheral nerves in vivo. PLoS Biology 11, e1001754 (2013).

14. Lewis, T. L., Jr., Turi, G. F., Kwon, S.-K., Losonczy, A. \& Polleux, F. Progressive Decrease of Mitochondrial Motility during Maturation of Cortical Axons In Vitro and In Vivo. Current Biology 26, 2602-2608 (2016).

15. Takihara, Y. et al. In vivo imaging of axonal transport of mitochondria in the diseased and aged mammalian CNS. Proceedings of the National Academy of Sciences 112, 10515-10520 (2015).

16. Zhou, B. et al. Facilitation of axon regeneration by enhancing mitochondrial transport and rescuing energy deficits. The Journal of Cell Biology 214, jcb.201605101-119 (2016).

17. Stowers, R. S., Megeath, L. J., Górska-Andrzejak, J., Meinertzhagen, I. A. \& Schwarz, T. L. Axonal transport of mitochondria to synapses depends on milton, a novel Drosophila protein. Neuron 36, 1063-1077 (2002).

18. van Spronsen, M. et al. TRAK/Milton motor-adaptor proteins steer mitochondrial trafficking to axons and dendrites. Neuron 77, 485-502 (2013).

19. Guo, X. et al. The GTPase dMiro is required for axonal transport of mitochondria to Drosophila synapses. Neuron 47, 379-393 (2005).

20. Russo, G. J. et al. Drosophila Miro is required for both anterograde and retrograde axonal mitochondrial transport. Journal of Neuroscience 29, 
5443-5455 (2009).

21. Babic, M. et al. Miro's N-terminal GTPase domain is required for transport of mitochondria into axons and dendrites. Journal of Neuroscience 35, 57545771 (2015).

22. MacAskill, A. F. et al. Miro1 is a calcium sensor for glutamate receptordependent localization of mitochondria at synapses. Neuron $61,541-555$ (2009).

23. Wang, X. \& Schwarz, T. L. The mechanism of Ca2+-dependent regulation of kinesin-mediated mitochondrial motility. Cell 136, 163-174 (2009).

24. Nguyen, T. T. et al. Loss of Miro1-directed mitochondrial movement results in a novel murine model for neuron disease. Proceedings of the National Academy of Sciences 111, E3631-40 (2014).

25. Lopez-Domenech, G. et al. Loss of Dendritic Complexity Precedes Neurodegeneration in a Mouse Model with Disrupted Mitochondrial Distribution in Mature Dendrites. Cell Rep 17, 317-327 (2016).

26. Chang, K. T., Niescier, R. F. \& Min, K.-T. Mitochondrial matrix Ca2+ as an intrinsic signal regulating mitochondrial motility in axons. Proceedings of the National Academy of Sciences 108, 15456-15461 (2011).

27. Misko, A., Jiang, S., Wegorzewska, I., Milbrandt, J. \& Baloh, R. H. Mitofusin 2 is necessary for transport of axonal mitochondria and interacts with the Miro/Milton complex. Journal of Neuroscience 30, 4232-4240 (2010).

28. Saotome, M. et al. Bidirectional Ca2+-dependent control of mitochondrial dynamics by the Miro GTPase. Proceedings of the National Academy of Sciences 105, 20728-20733 (2008).

29. MacAskill, A. F., Brickley, K., Stephenson, F. A. \& Kittler, J. T. GTPase dependent recruitment of Grif-1 by Miro1 regulates mitochondrial trafficking in hippocampal neurons. Mol. Cell. Neurosci. 40, 301-312 (2009).

30. Pekkurnaz, G., Trinidad, J. C., Wang, X., Kong, D. \& Schwarz, T. L. Glucose regulates mitochondrial motility via Milton modification by O-GIcNAc transferase. Cell 158, 54-68 (2014).

31. Ferreira, J. M., Burnett, A. L. \& Rameau, G. A. Activity-dependent regulation of surface glucose transporter-3. Journal of Neuroscience 31, 1991-1999 (2011).

32. Ashrafi, G., Wu, Z., Farrell, R. J. \& Ryan, T. A. GLUT4 Mobilization Supports Energetic Demands of Active Synapses. Neuron (2017). doi:10.1016/j.neuron.2016.12.020

33. Perkins, G. A. et al. The micro-architecture of mitochondria at active zones: electron tomography reveals novel anchoring scaffolds and cristae structured for high-rate metabolism. Journal of Neuroscience 30, 1015-1026 (2010).

34. Davey, G. P., Peuchen, S. \& Clark, J. B. Energy thresholds in brain mitochondria potential involvement in neurodegeneration. Journal of Biological Chemistry 273, 12753-12757 (1998).

35. Brown, M. R., Sullivan, P. G. \& Geddes, J. W. Synaptic mitochondria are more susceptible to Ca2+overload than nonsynaptic mitochondria. The Journal of biological chemistry 281, 11658-11668 (2006).

36. Rangaraju, V., Dieck, S. T. \& Schuman, E. M. Local translation in neuronal compartments: how local is local? EMBO Rep 18, 693-711 (2017).

37. Spillane, M., Ketschek, A., Merianda, T. T., Twiss, J. L. \& Gallo, G. Mitochondria Coordinate Sites of Axon Branching through Localized Intraaxonal Protein Synthesis. Cell Rep 5, 1564-1575 (2013).

38. Meyer, M. P. \& Smith, S. J. Evidence from in vivo imaging that synaptogenesis guides the growth and branching of axonal arbors by two 
distinct mechanisms. Journal of Neuroscience 26, 3604-3614 (2006).

39. Ruthazer, E. S., Li, J. \& Cline, H. T. Stabilization of Axon Branch Dynamics by Synaptic Maturation. J Neurosci 26, 3594-3603 (2006).

40. Courchet, J. et al. Terminal axon branching is regulated by the LKB1-NUAK1 kinase pathway via presynaptic mitochondrial capture. Cell 153, 1510-1525 (2013).

41. Lee, C. W. \& Peng, H. B. The function of mitochondria in presynaptic development at the neuromuscular junction. Mol. Biol. Cell 19, 150-158 (2008).

42. Smirnova, E., Shurland, D. L., Ryazantsev, S. N. \& van der Bliek, A. M. A human dynamin-related protein controls the distribution of mitochondria. The Journal of Cell Biology 143, 351-358 (1998).

43. $\quad \mathrm{Li}, \mathrm{H}$. et al. Bcl-xL induces Drp1-dependent synapse formation in cultured hippocampal neurons. Proceedings of the National Academy of Sciences 105, 2169-2174 (2008).

44. Huttenlocher, P. R., de Courten, C., Garey, L. J. \& Van der Loos, H. Synaptogenesis in human visual cortex - evidence for synapse elimination during normal development. Neuroscience Letters 33, 247-252 (1982).

45. Walsh, M. K. \& Lichtman, J. W. In vivo time-lapse imaging of synaptic takeover associated with naturally occurring synapse elimination. Neuron (2003).

46. Zuo, Y., Lin, A., Chang, P. \& Gan, W.-B. Development of long-term dendritic spine stability in diverse regions of cerebral cortex. Neuron 46, 181-189 (2005).

47. Wiesel, T. N. Postnatal development of the visual cortex and the influence of environment. Nature 299, 583-591 (1982).

48. Colman, H., Nabekura, J. \& Lichtman, J. W. Alterations in Synaptic Strength Preceding Axon Withdrawal. Science 275, 356-361 (1997).

49. Chen, C. \& Regehr, W. G. Developmental Remodeling of the Retinogeniculate Synapse. Neuron 28, 955-966 (2000).

50. Buffelli, M. et al. Genetic evidence that relative synaptic efficacy biases the outcome of synaptic competition. Nature 424, 430-434 (2003).

51. Li, Z., Okamoto, K.-I., Hayashi, Y. \& Sheng, M. The Importance of Dendritic Mitochondria in the Morphogenesis and Plasticity of Spines and Synapses. Cell 119, 873-887 (2004).

52. Ertürk, A., Wang, Y. \& Sheng, M. Local Pruning of Dendrites and Spines by Caspase-3-Dependent and Proteasome-Limited Mechanisms. J Neurosci 34, 1672-1688 (2014).

53. Meng, L. et al. The Cell Death Pathway Regulates Synapse Elimination through Cleavage of Gelsolin in Caenorhabditis elegans Neurons. Cell Rep 11, 1737-1748 (2015).

54. Zala, D. et al. Vesicular glycolysis provides on-board energy for fast axonal transport. Cell 152, 479-491 (2013).

55. Nehlig, A. Brain uptake and metabolism of ketone bodies in animal models. Prostaglandins, Leukotrienes and Essential Fatty Acids 70, 265-275 (2004).

56. Fukao, T., Lopaschuk, G. D. \& Mitchell, G. A. Pathways and control of ketone body metabolism: on the fringe of lipid biochemistry. Prostaglandins, Leukotrienes and Essential Fatty Acids 70, 243-251 (2004).

57. Zheng, X. et al. Metabolic reprogramming during neuronal differentiation from aerobic glycolysis to neuronal oxidative phosphorylation. Elife 5, e13374 (2016).

58. Hall, C. N., Klein-Flügge, M. C., Howarth, C. \& Attwell, D. Oxidative 
phosphorylation, not glycolysis, powers presynaptic and postsynaptic mechanisms underlying brain information processing. Journal of Neuroscience 32, 8940-8951 (2012).

This study demonstrates that oxidative phosphorylation is central for powering synaptic transmission.

59. Harris, J. J., Jolivet, R. \& Attwell, D. Synaptic Energy Use and Supply. Neuron 75, 762-777 (2012).

60. Liotta, A. et al. Energy demand of synaptic transmission at the hippocampal Schaffer-collateral synapse. J. Cereb. Blood Flow Metab. 32, 2076-2083 (2012).

61. Alnaes, E. \& Rahamimoff, R. On the role of mitochondria in transmitter release from motor nerve terminals. J. Physiol. (Lond.) 248, 285-306 (1975).

This is an early demonstration that inhibiting oxidative phosphorylation compromises synaptic transmission during prolonged activity.

62. Verstreken, P. et al. Synaptic mitochondria are critical for mobilization of reserve pool vesicles at Drosophila neuromuscular junctions. Neuron 47, 365-378 (2005).

This study provides genetic evidence from a fly model that depleting presynapses of mitochondria leads to failure of prolonged synaptic activity.

63. Bi, G. Q. et al. Kinesin- and myosin-driven steps of vesicle recruitment for Ca2+-regulated exocytosis. The Journal of Cell Biology 138, 999-1008 (1997).

64. Boissan, M. et al. Membrane trafficking. Nucleoside diphosphate kinases fuel dynamin superfamily proteins with GTP for membrane remodeling. Science 344, 1510-1515 (2014).

65. Ishihara, N. et al. Mitochondrial fission factor Drp1 is essential for embryonic development and synapse formation in mice. Nat Cell Biol 11, 958-966 (2009).

66. Berthet, A. et al. Loss of mitochondrial fission depletes axonal mitochondria in midbrain dopamine neurons. Journal of Neuroscience 34, 14304-14317 (2014).

67. Shields, L. Y. et al. Dynamin-related protein 1 is required for normal mitochondrial bioenergetic and synaptic function in CA1 hippocampal neurons. Cell Death Dis 6, e1725 (2015).

68. Oettinghaus, B. et al. Synaptic dysfunction, memory deficits and hippocampal atrophy due to ablation of mitochondrial fission in adult forebrain neurons. Cell death and differentiation 23, 18-28 (2016).

69. Cai, Q., Gerwin, C. \& Sheng, Z.-H. Syntabulin-mediated anterograde transport of mitochondria along neuronal processes. The Journal of Cell Biology 170, 959-969 (2005).

70. Ma, H., Cai, Q., Lu, W., Sheng, Z.-H. \& Mochida, S. KIF5B motor adaptor syntabulin maintains synaptic transmission in sympathetic neurons. Journal of Neuroscience 29, 13019-13029 (2009).

71. Rangaraju, V., Calloway, N. \& Ryan, T. A. Activity-driven local ATP synthesis is required for synaptic function. Cell 156, 825-835 (2014).

This study demonstrated that local synthesis of ATP (via glycolysis and oxidative phosphorylation) is prerequisite for synaptic function.

72. Jang, S. et al. Glycolytic Enzymes Localize to Synapses under Energy Stress to Support Synaptic Function. Neuron 90, 278-291 (2016).

73. Zador, A. Impact of Synaptic Unreliability on the Information Transmitted by Spiking Neurons. Journal of Neurophysiology 79, 1219-1229 (1998). 
74. Sun, T., Qiao, H., Pan, P.-Y., Chen, Y. \& Sheng, Z.-H. Motile axonal mitochondria contribute to the variability of presynaptic strength. Cell Rep 4, 413-419 (2013).

This study provides evidence that increasing movement of mitochondria to and from presynapses increases the variability of synaptic transmission.

75. Pathak, D. et al. The role of mitochondrially derived ATP in synaptic vesicle recycling. Journal of Biological Chemistry 290, 22325-22336 (2015).

76. Belles, B., Hescheler, J. \& Trube, G. Changes of membrane currents in cardiac cells induced by long whole-cell recordings and tolbutamide. Pflugers Arch. 409, 582-588 (1987).

77. Hubley, M. J., Locke, B. R. \& Moerland, T. S. The effects of temperature, pH, and magnesium on the diffusion coefficient of ATP in solutions of physiological ionic strength. Biochimica et Biophysica Acta (BBA) - General Subjects 1291, 115-121 (1996).

78. Katz, B. \& Miledi, R. The role of calcium in neuromuscular facilitation. $J$. Physiol. (Lond.) 195, 481-492 (1968).

79. Mulkey, R. M. \& Zucker, R. S. Posttetanic potentiation at the crayfish neuromuscular junction is dependent on both intracellular calcium and sodium ion accumulation. J Neurosci 12, 4327-4336 (1992).

80. Tang, Y. \& Zucker, R. S. Mitochondrial involvement in post-tetanic potentiation of synaptic transmission. Neuron 18, 483-491 (1997).

This study suggests that mitochondria could, under conditions of intense stimulation, prolongue the elevation in presynaptic $\mathrm{Ca}^{2+}$, thereby contributing to post tentanic potentiation.

81. David, G., Barrett, J. N. \& Barrett, E. F. Evidence that mitochondria buffer physiological $\mathrm{Ca} 2+$ loads in lizard motor nerve terminals. J. Physiol. (Lond.) 509, 59-65 (1998).

This study suggests that, under physiological stimulation conditions, mitochondria slow down the elevation in presynaptic $\mathrm{Ca}^{2+}$.

82. David, G. \& Barrett, E. F. Stimulation-evoked increases in cytosolic [Ca(2+)] in mouse motor nerve terminals are limited by mitochondrial uptake and are temperature-dependent. Journal of Neuroscience 20, 7290-7296 (2000).

83. Chouhan, A. K., Zhang, J., Zinsmaier, K. E. \& Macleod, G. T. Presynaptic mitochondria in functionally different motor neurons exhibit similar affinities for $\mathrm{Ca} 2+$ but exert little influence as $\mathrm{Ca} 2+$ buffers at nerve firing rates in situ. Journal of Neuroscience 30, 1869-1881 (2010).

84. Billups, B. \& Forsythe, I. D. Presynaptic mitochondrial calcium sequestration influences transmission at mammalian central synapses. Journal of Neuroscience 22, 5840-5847 (2002).

This study shows that mitochondria at the calyx of Held slow removal of $\mathrm{Ca}^{2+}$, but this lowers neurotransmission, possibly by altering the balance between several $\mathrm{Ca}^{2+}$-mediated processes that regulate neurotransmission.

85. Kim, M.-H., Korogod, N., Schneggenburger, R., Ho, W.-K. \& Lee, S.-H. Interplay between $\mathrm{Na}+\mathrm{Ca} 2+$ exchangers and mitochondria in $\mathrm{Ca} 2+$ clearance at the calyx of Held. Journal of Neuroscience 25, 6057-6065 (2005).

86. Waters, J. \& Smith, S. J. Mitochondria and release at hippocampal synapses. Pflugers Arch. 447, 363-370 (2003).

87. Zenisek, D. \& Matthews, G. The role of mitochondria in presynaptic calcium handling at a ribbon synapse. Neuron 25, 229-237 (2000). 
88. Levy, M., Faas, G. C., Saggau, P., Craigen, W. J. \& Sweatt, J. D. Mitochondrial regulation of synaptic plasticity in the hippocampus. The Journal of biological chemistry 278, 17727-17734 (2003).

89. Molgo, J. \& Pecot-Dechavassine, M. Effects of carbonyl cyanide mchlorophenylhydrazone on quantal transmitter release and ultrastructure of frog motor nerve terminals. Neuroscience 24, 695-708 (1988).

90. Zengel, J. E., Sosa, M. A., Poage, R. E. \& Mosier, D. R. Role of intracellular $\mathrm{Ca2}+$ in stimulation-induced increases in transmitter release at the frog neuromuscular junction. J Gen Physiol 104, 337-355 (1994).

91. Calupca, M. A., Prior, C., Merriam, L. A., Hendricks, G. M. \& Parsons, R. L. Presynaptic function is altered in snake $\mathrm{K}+$-depolarized motor nerve terminals containing compromised mitochondria. J. Physiol. (Lond.) 532, 217-227 (2001).

92. David, G. \& Barrett, E. F. Mitochondrial Ca2+ uptake prevents desynchronization of quantal release and minimizes depletion during repetitive stimulation of mouse motor nerve terminals. J. Physiol. (Lond.) 548, 425-438 (2003).

93. Gazit, N. et al. IGF-1 Receptor Differentially Regulates Spontaneous and Evoked Transmission via Mitochondria at Hippocampal Synapses. Neuron 89, 583-597 (2016).

This study shows that IGF-1 signalling can simultaneously modulate both evoked and spontaneous synaptic transmission, by acting on mitochondrial ATP production and $\mathrm{Ca}^{2+}$ buffering.

94. Kamer, K. J. \& Mootha, V. K. The molecular era of the mitochondrial calcium uniporter. Nature Reviews Molecular Cell Biology 16, 545-553 (2015).

95. Shutov, L. P., Kim, M.-S., Houlihan, P. R., Medvedeva, Y. V. \& Usachev, Y. M. Mitochondria and plasma membrane Ca2+-ATPase control presynaptic $\mathrm{Ca2}+$ clearance in capsaicin-sensitive rat sensory neurons. J. Physiol. (Lond.) 591, 2443-2462 (2013).

This study finds that presynaptic mitochondria account for around $40 \%$ of $\mathrm{Ca}^{2+}$ clearance in rat sensory neurons.

96. Marland, J. R. K., Hasel, P., Bonnycastle, K. \& Cousin, M. A. Mitochondrial Calcium Uptake Modulates Synaptic Vesicle Endocytosis in Central Nerve Terminals. Journal of Biological Chemistry 291, 2080-2086 (2016).

97. Vaccaro, V., Devine, M. J., Higgs, N. F. \& Kittler, J. T. Miro1-dependent mitochondrial positioning drives the rescaling of presynaptic $\mathrm{Ca} 2+$ signals during homeostatic plasticity. EMBO Rep 18, 231-240 (2017).

This study demonstrates that presynaptic mitochondria buffer $\mathrm{Ca}^{2+}$ signals via MCU, thereby downregulating neurotransmission, and mitochondria are recruited to and from presynapses in response to long term changes in network activity.

98. Kwon, S.-K. et al. LKB1 Regulates Mitochondria-Dependent Presynaptic Calcium Clearance and Neurotransmitter Release Properties at Excitatory Synapses along Cortical Axons. PLoS Biology 14, e1002516 (2016).

This study demonstrates that presynaptic mitochondria buffer $\mathrm{Ca}^{2+}$ via $\mathrm{MCU}$, thus lowering neurotransmission, and $\mathrm{MCU}$ abundance is regulated by LKB1.

99. Kim, H. Y. et al. Mitochondrial $\mathrm{Ca}(2+)$ uptake is essential for synaptic plasticity in pain. Journal of Neuroscience 31, 12982-12991 (2011).

100. Dobrunz, L. E. \& Stevens, C. F. Heterogeneity of Release Probability, Facilitation, and Depletion at Central Synapses. Neuron 18, 995-1008 (1997). 
101. Duchen, M. R. Ca(2+)-dependent changes in the mitochondrial energetics in single dissociated mouse sensory neurons. Biochem J 283 ( Pt 1), 41-50 (1992).

102. Luongo, T. S. et al. The Mitochondrial Calcium Uniporter Matches Energetic Supply with Cardiac Workload during Stress and Modulates Permeability Transition. Cell Rep 12, 23-34 (2015).

103. Llorente Folch, I. et al. The regulation of neuronal mitochondrial metabolism by calcium. J. Physiol. (Lond.) 593, 3447-3462 (2015).

104. Paillusson, S. et al. There's Something Wrong with my MAM; the ERMitochondria Axis and Neurodegenerative Diseases. Trends in Neurosciences 39, 146-157 (2016).

105. Wu, Y. et al. Contacts between the endoplasmic reticulum and other membranes in neurons. Proceedings of the National Academy of Sciences 114, E4859-E4867 (2017).

This ultrastructural study shows that ER forms a web around mitochondria and other organelles at presynapses.

106. Mironov, S. L. \& Symonchuk, N. ER vesicles and mitochondria move and communicate at synapses. Journal of Cell Science 119, 4926-4934 (2006).

107. Csordás, G. et al. Imaging Interorganelle Contacts and Local Calcium Dynamics at the ER-Mitochondrial Interface. Molecular Cell 39, 121-132 (2010).

This study demonstrates that pockets of high [Ca2+] exist at sites of contact between ER and mitochondria.

108. Lee, S. et al. Polo Kinase Phosphorylates Miro to Control ER-Mitochondria Contact Sites and Mitochondrial Ca2+ Homeostasis in Neural Stem Cell Development. Dev. Cell 37, 174-189 (2016).

109. Kornmann, B., Osman, C. \& Walter, P. The conserved GTPase Gem1 regulates endoplasmic reticulum-mitochondria connections. Proceedings of the National Academy of Sciences 108, 14151-14156 (2011).

110. de Juan-Sanz, J. et al. Axonal Endoplasmic Reticulum Ca2+ Content Controls Release Probability in CNS Nerve Terminals. Neuron 0, 867881.e6 (2017).

This study demonstrates that ER also have a role in regulating neurotransmission via modulating presynaptic $\mathrm{Ca}^{2+}$ signals.

111. Butow, R. A. \& Avadhani, N. G. Mitochondrial Signaling. Molecular Cell 14, $1-15(2004)$.

112. Cagin, U. et al. Mitochondrial retrograde signaling regulates neuronal function. Proceedings of the National Academy of Sciences 112, E6000-9 (2015).

113. Accardi, M. V. et al. Mitochondrial reactive oxygen species regulate the strength of inhibitory GABA-mediated synaptic transmission. Nature Communications 5, (2014).

114. Sena, L. A. \& Chandel, N. S. Physiological Roles of Mitochondrial Reactive Oxygen Species. Molecular Cell 48, 158-167 (2012).

115. Su, B., Ji, Y. S., Sun, X. L., Liu, X. H. \& Chen, Z. Y. Brain-derived neurotrophic factor (BDNF)-induced mitochondrial motility arrest and presynaptic docking contribute to BDNF-enhanced synaptic transmi... PubMed - NCBI. The Journal of biological chemistry 289, 1213-1226 (2014).

116. Bénard, G. et al. Mitochondrial CB1 receptors regulate neuronal energy metabolism. Nat Neurosci 15, 558-564 (2012).

117. Hebert-Chatelain, E. et al. A cannabinoid link between mitochondria and memory. Nature 539, 555-559 (2016). 
118. Sibson, N. R. et al. Stoichiometric coupling of brain glucose metabolism and glutamatergic neuronal activity. Proceedings of the National Academy of Sciences of the United States of America 95, 316-321 (1998).

119. Waagepetersen, H. S., Sonnewald, U., Gegelashvili, G., Larsson, O. M. \& Schousboe, A. Metabolic distinction between vesicular and cytosolic GABA in cultured GABAergic neurons using $13 \mathrm{C}$ magnetic resonance spectroscopy. J Neurosci Res 63, 347-355 (2001).

120. Sandoval, H. et al. Mitochondrial fusion but not fission regulates larval growth and synaptic development through steroid hormone production. Elife 3, (2014).

121. Remage-Healey, L., Saldanha, C. J. \& Schlinger, B. A. Estradiol Synthesis and Action at the Synapse: Evidence for 'Synaptocrine' Signaling. Front. Endocrinol. 2, (2011).

122. Sarzi, E. et al. Increased steroidogenesis promotes early-onset and severe vision loss in females with OPA1 dominant optic atrophy. Human Molecular Genetics 25, 2539-2551 (2016).

123. Chernova, T., Nicotera, P. \& Smith, A. G. Heme Deficiency Is Associated with Senescence and Causes Suppression of N-Methyl-d-aspartate Receptor Subunits Expression in Primary Cortical Neurons. Mol Pharmacol 69, 697-705 (2006).

124. Chernova, T. et al. Neurite degeneration induced by heme deficiency mediated via inhibition of NMDA receptor-dependent extracellular signalregulated kinase 1/2 activation. Journal of Neuroscience 27, 8475-8485 (2007).

125. Shidara, Y. \& Hollenbeck, P. J. Defects in mitochondrial axonal transport and membrane potential without increased reactive oxygen species production in a Drosophila model of Friedreich ataxia. Journal of Neuroscience 30, 1136911378 (2010).

126. Bissell, D. M., Anderson, K. E. \& Bonkovsky, H. L. Porphyria. N Engl J Med 377, 862-872 (2017).

127. Varela, L., Schwartz, M. L. \& Horvath, T. L. Mitochondria controlled by UCP2 determine hypoxia-induced synaptic remodeling in the cortex and hippocampus. Neurobiology of Disease (2016). doi:10.1016/j.nbd.2016.01.004

128. Herrup, K. \& Yang, Y. Cell cycle regulation in the postmitotic neuron: oxymoron or new biology? Nat Rev Neurosci 8, 368-378 (2007).

129. Ferreirinha, F. et al. Axonal degeneration in paraplegin-deficient mice is associated with abnormal mitochondria and impairment of axonal transport. J. Clin. Invest. 113, 231-242 (2004).

130. Scheff, S. W., Price, D. A., Schmitt, F. A., DeKosky, S. T. \& Mufson, E. J. Synaptic alterations in CA1 in mild Alzheimer disease and mild cognitive impairment. Neurology 68, 1501-1508 (2007).

131. Cheng, H. C., Ulane, C. M. \& Burke, R. E. Clinical progression in Parkinson disease and the neurobiology of axons. Ann Neurol 67, 715-725 (2010).

132. Hardy, J. Amyloid double trouble. Nature Genetics 38, 11-12 (2006).

133. Keller, J. N. et al. Impairment of Glucose and Glutamate Transport and Induction of Mitochondrial Oxidative Stress and Dysfunction in Synaptosomes by Amyloid $\beta$-Peptide: Role of the Lipid Peroxidation Product 4-Hydroxynonenal. Journal of neurochemistry 69, 273-284 (1997).

134. Keller, J. N. et al. Amyloid $\beta$-Peptide Effects on Synaptosomes from Apolipoprotein E-Deficient Mice. Journal of neurochemistry 74, 1579-1586 (2000). 
135. Mungarro Menchaca, X., Ferrera, P., Morán, J. \& Arias, C. $\beta$-Amyloid peptide induces ultrastructural changes in synaptosomes and potentiates mitochondrial dysfunction in the presence of ryanodine. J Neurosci Res 68, 89-96 (2002).

136. Rui, Y., Tiwari, P., Xie, Z. \& Zheng, J. Q. Acute impairment of mitochondrial trafficking by beta-amyloid peptides in hippocampal neurons. Journal of Neuroscience 26, 10480-10487 (2006).

137. Wang, X., Perry, G., Smith, M. A. \& Zhu, X. Amyloid-beta-derived diffusible ligands cause impaired axonal transport of mitochondria in neurons.

Neurodegenerative Dis 7, 56-59 (2010).

138. Lin, M.-Y. et al. Releasing Syntaphilin Removes Stressed Mitochondria from Axons Independent of Mitophagy under Pathophysiological Conditions.

Neuron 94, 595-610.e6 (2017).

139. Ye, X., Sun, X., Starovoytov, V. \& Cai, Q. Parkin-mediated mitophagy in mutant hAPP neurons and Alzheimer's disease patient brains. Human Molecular Genetics 24, 2938-2951 (2015).

140. Cho, D.-H. et al. S-Nitrosylation of Drp1 Mediates $\beta$-Amyloid-Related Mitochondrial Fission and Neuronal Injury. Science 324, 102-105 (2009).

141. Manczak, M., Calkins, M. J. \& Reddy, P. H. Impaired mitochondrial dynamics and abnormal interaction of amyloid beta with mitochondrial protein Drp1 in neurons from patients with Alzheimer's disease: implications for neuronal damage. Human Molecular Genetics 20, 2495-2509 (2011).

142. Manczak, M., Kandimalla, R., Fry, D., Sesaki, H. \& Reddy, P. H. Protective effects of reduced dynamin-related protein 1 against amyloid beta-induced mitochondrial dysfunction and synaptic damage in Alzheimer's disease. Human Molecular Genetics 25, 5148-5166 (2016).

143. DuBoff, B., Götz, J. \& Feany, M. B. Tau Promotes Neurodegeneration via DRP1 Mislocalization In Vivo. Neuron 75, 618-632 (2012).

This study shows that disease-associated mutant tau inhibits DRP1 activity leading to impaired mitochondrial fission.

144. Kandimalla, R. et al. Reduced dynamin-related protein 1 protects against phosphorylated Tau-induced mitochondrial dysfunction and synaptic damage in Alzheimer's disease. Human Molecular Genetics 25, 4881-4897 (2016).

145. Shahpasand, K. et al. Regulation of mitochondrial transport and intermicrotubule spacing by tau phosphorylation at the sites hyperphosphorylated in Alzheimer's disease. Journal of Neuroscience 32, 2430-2441 (2012).

146. lijima-Ando, K. et al. Loss of axonal mitochondria promotes tau-mediated neurodegeneration and Alzheimer's disease-related tau phosphorylation via PAR-1. PLoS Genetics 8, e1002918 (2012).

147. Roberson, E. D. et al. Reducing Endogenous Tau Ameliorates Amyloid BInduced Deficits in an Alzheimer's Disease Mouse Model. Science 316, 750754 (2007).

148. Ittner, L. M. et al. Dendritic Function of Tau Mediates Amyloid- $\beta$ Toxicity in Alzheimer's Disease Mouse Models. Cell 142, 387-397 (2010).

149. Vossel, K. A. et al. Tau reduction prevents Abeta-induced defects in axonal transport. Science 330, 198-198 (2010).

150. Hansson Petersen, C. A. et al. The amyloid beta-peptide is imported into mitochondria via the TOM import machinery and localized to mitochondrial cristae. Proceedings of the National Academy of Sciences 105, 1314513150 (2008).

151. Du, H. et al. Cyclophilin D deficiency attenuates mitochondrial and neuronal perturbation and ameliorates learning and memory in Alzheimer's disease. 
Nature Medicine 14, 1097-1105 (2008).

152. Zhao, X.-L. et al. Expression of beta-amyloid induced age-dependent presynaptic and axonal changes in Drosophila. Journal of Neuroscience 30, 1512-1522 (2010).

153. Lee, S.-H. et al. Impaired short-term plasticity in mossy fiber synapses caused by mitochondrial dysfunction of dentate granule cells is the earliest synaptic deficit in a mouse model of Alzheimer's disease. Journal of Neuroscience 32, 5953-5963 (2012).

This study shows that presynaptic mitochondrial handling of $\mathrm{Ca}^{2+}$ is altered in an AD mouse model.

154. Lee, S.-H. et al. Presenilins regulate synaptic plasticity and mitochondrial calcium homeostasis in the hippocampal mossy fiber pathway. Mol Neurodegener 12, 48 (2017).

155. Moloney, A. M. et al. Defects in IGF-1 receptor, insulin receptor and IRS-1/2 in Alzheimer's disease indicate possible resistance to IGF-1 and insulin signalling. Neurobiology of aging 31, 224-243 (2010).

156. Zhang, B., Tang, X. C. \& Zhang, H. Y. Alternations of central insulin-like growth factor-1 sensitivity in APP/PS1 transgenic mice and neuronal models. J Neurosci Res 91, 717-725 (2013).

157. Hedskog, L. et al. Modulation of the endoplasmic reticulum-mitochondria interface in Alzheimer's disease and related models. Proceedings of the National Academy of Sciences 110, 7916-7921 (2013).

This study shows that MAM proteins are upregulated in brain tissue from people with $A D$ and in an AD mouse model.

158. Schapira, A. H. V. et al. MITOCHONDRIAL COMPLEX I DEFICIENCY IN PARKINSON'S DISEASE. The Lancet 333, 1269 (1989).

159. Valente, E. M. Hereditary Early-Onset Parkinson's Disease Caused by Mutations in PINK1. Science 304, 1158-1160 (2004).

160. Kitada, T. et al. Mutations in the parkin gene cause autosomal recessive juvenile parkinsonism. Nature 392, 605-608 (1998).

161. Miller, K. E. \& Sheetz, M. P. Axonal mitochondrial transport and potential are correlated. Journal of Cell Science 117, 2791-2804 (2004).

162. Cai, Q., Zakaria, H. M., Simone, A. \& Sheng, Z.-H. Spatial Parkin Translocation and Degradation of Damaged Mitochondria via Mitophagy in Live Cortical Neurons. Current Biology 22, 545-552 (2012).

163. Devireddy, S., Liu, A., Lampe, T. \& Hollenbeck, P. J. The Organization of Mitochondrial Quality Control and Life Cycle in the Nervous System In Vivo in the Absence of PINK1. Journal of Neuroscience 35, 9391-9401 (2015).

164. Sung, H., Tandarich, L. C., Nguyen, K. \& Hollenbeck, P. J. Compartmentalized Regulation of Parkin-Mediated Mitochondrial Quality Control in the Drosophila Nervous System In Vivo. Journal of Neuroscience 36, 7375-7391 (2016).

165. Sterky, F. H., Lee, S., Wibom, R., Olson, L. \& Larsson, N.-G. Impaired mitochondrial transport and Parkin-independent degeneration of respiratory chain-deficient dopamine neurons in vivo. Proceedings of the National Academy of Sciences 108, 12937-12942 (2011).

166. Kim-Han, J. S., Antenor-Dorsey, J. A. \& O'Malley, K. L. The parkinsonian mimetic, MPP+, specifically impairs mitochondrial transport in dopamine axons. Journal of Neuroscience 31, 7212-7221 (2011).

167. Dukes, A. A. et al. Live imaging of mitochondrial dynamics in CNS dopaminergic neurons in vivo demonstrates early reversal of mitochondrial transport following MPP+ exposure. Neurobiology of Disease 95, 238-249 
(2016).

168. Wang, X. et al. PINK1 and Parkin Target Miro for Phosphorylation and Degradation to Arrest Mitochondrial Motility. Cell 147, 893-906 (2011).

169. Birsa, N. et al. Lysine 27 ubiquitination of the mitochondrial transport protein Miro is dependent on serine 65 of the Parkin ubiquitin ligase. Journal of Biological Chemistry 289, 14569-14582 (2014).

170. Hsieh, C.-H. et al. Functional Impairment in Miro Degradation and Mitophagy Is a Shared Feature in Familial and Sporadic Parkinson's Disease. Cell Stem Cell 19, 709-724 (2016).

171. Ashrafi, G., Schlehe, J. S., Lavoie, M. J. \& Schwarz, T. L. Mitophagy of damaged mitochondria occurs locally in distal neuronal axons and requires PINK1 and Parkin. The Journal of Cell Biology 206, 655-670 (2014).

This study suggests that mitophagy can occur locally in distal axons without needing to transport damaged mitochondria back to the soma.

172. Kitada, T. et al. Impaired dopamine release and synaptic plasticity in the striatum of PINK1-deficient mice. Proceedings of the National Academy of Sciences of the United States of America 104, 11441-11446 (2007).

173. Morais, V. A. et al. Parkinson's disease mutations in PINK1 result in decreased Complex I activity and deficient synaptic function. EMBO Mol Med 1, 99-111 (2009).

174. Polymeropoulos, M. H. et al. Mutation in the alpha-synuclein gene identified in families with Parkinson's disease. Science 276, 2045-2047 (1997).

175. Spillantini, M. G. et al. Alpha-synuclein in Lewy bodies. Nature 388, 839-840 (1997).

176. Cole, N. B., Dieuliis, D., Leo, P., Mitchell, D. C. \& Nussbaum, R. L. Mitochondrial translocation of alpha-synuclein is promoted by intracellular acidification. Experimental Cell Research 314, 2076-2089 (2008).

177. Hsu, L. J. et al. alpha-synuclein promotes mitochondrial deficit and oxidative stress. The American journal of pathology 157, 401-410 (2000).

178. Martin, L. J. et al. Parkinson's disease alpha-synuclein transgenic mice develop neuronal mitochondrial degeneration and cell death. Journal of Neuroscience 26, 41-50 (2006).

179. Li, L. et al. Human A53T a-synuclein causes reversible deficits in mitochondrial function and dynamics in primary mouse cortical neurons. PLoS One 8, e85815 (2013).

180. Kramer, M. L. \& Schulz-Schaeffer, W. J. Presynaptic alpha-synuclein aggregates, not Lewy bodies, cause neurodegeneration in dementia with Lewy bodies. Journal of Neuroscience 27, 1405-1410 (2007).

181. Nemani, V. M. et al. Increased expression of alpha-synuclein reduces neurotransmitter release by inhibiting synaptic vesicle reclustering after endocytosis. Neuron 65, 66-79 (2010).

182. Scott, D. A. et al. A pathologic cascade leading to synaptic dysfunction in alpha-synuclein-induced neurodegeneration. Journal of Neuroscience 30, 8083-8095 (2010).

183. Guardia-Laguarta, C. et al. a-Synuclein is localized to mitochondriaassociated ER membranes. Journal of Neuroscience 34, 249-259 (2014).

184. Paillusson, S. et al. a-Synuclein binds to the ER-mitochondria tethering protein VAPB to disrupt $\mathrm{Ca} 2+$ homeostasis and mitochondrial ATP production. Acta Neuropathol 28, 1-21 (2017).

185. De Vos, K. J. et al. VAPB interacts with the mitochondrial protein PTPIP51 to regulate calcium homeostasis. Human Molecular Genetics 21, 1299-1311 (2012). 
186. Gautier, C. A. et al. The endoplasmic reticulum-mitochondria interface is perturbed in PARK2 knockout mice and patients with PARK2 mutations. Human Molecular Genetics 25, 2972-2984 (2016).

187. Rosen, D. R. et al. Mutations in $\mathrm{Cu} / \mathrm{Zn}$ superoxide dismutase gene are associated with familial amyotrophic lateral sclerosis. Nature 362, 362059a0-62 (1993).

188. De Vos, K. J. et al. Familial amyotrophic lateral sclerosis-linked SOD1 mutants perturb fast axonal transport to reduce axonal mitochondria content. Human Molecular Genetics 16, 2720-2728 (2007).

189. Moller, A., Bauer, C. S., Cohen, R. N., Webster, C. P. \& De Vos, K. J. Amyotrophic lateral sclerosis-associated mutant SOD1 inhibits anterograde axonal transport of mitochondria by reducing Miro1 levels. Human Molecular Genetics (2017). doi:10.1093/hmg/ddx348

190. Baldwin, K. R., Godena, V. K., Hewitt, V. L. \& Whitworth, A. J. Axonal transport defects are a common phenotype in Drosophila models of ALS. Human Molecular Genetics 25, 2378-2392 (2016).

191. Mórotz, G. M. et al. Amyotrophic lateral sclerosis-associated mutant VAPBP56S perturbs calcium homeostasis to disrupt axonal transport of mitochondria. Human Molecular Genetics 21, 1979-1988 (2012).

192. Stoica, R. et al. ER-mitochondria associations are regulated by the VAPBPTPIP51 interaction and are disrupted by ALS/FTD-associated TDP-43. Nature Communications 5, (2014).

193. Neumann, M. et al. Ubiquitinated TDP-43 in frontotemporal lobar degeneration and amyotrophic lateral sclerosis. Science 314, 130-133 (2006).

194. Sreedharan, J. et al. TDP-43 Mutations in Familial and Sporadic Amyotrophic Lateral Sclerosis. Science 319, 1668-1672 (2008).

195. Covill-Cooke, C., Howden, J. H., Birsa, N. \& Kittler, J. T. Ubiquitination at the mitochondria in neuronal health and disease. Neurochemistry International (2017). doi:10.1016/j.neuint.2017.07.003

196. McLelland, G. L., Soubannier, V., Chen, C. X., McBride, H. M. \& Fon, E. A. Parkin and PINK1 function in a vesicular trafficking pathway regulating mitochondrial quality control. The EMBO Journal 33, 282-295 (2014).

197. Neuspiel, M. et al. Cargo-Selected Transport from the Mitochondria to Peroxisomes Is Mediated by Vesicular Carriers. Current Biology 18, 102-108 (2008).

198. Bhujabal, Z. et al. FKBP8 recruits LC3A to mediate Parkin-independent mitophagy. EMBO Rep 18, 947-961 (2017).

199. Liu, L. et al. Mitochondrial outer-membrane protein FUNDC1 mediates hypoxia-induced mitophagy in mammalian cells. Nat Cell Biol 14, 177-185 (2012).

200. Chen, G. et al. A Regulatory Signaling Loop Comprising the PGAM5 Phosphatase and CK2 Controls Receptor-Mediated Mitophagy. Molecular Cell 54, 362-377 (2014).

201. Davis, C.-H. O. et al. Transcellular degradation of axonal mitochondria. Proceedings of the National Academy of Sciences 111, 9633-9638 (2014).

202. Wood, J. G. et al. Sirtuin activators mimic caloric restriction and delay ageing in metazoans. Nature 430, 686-689 (2004).

203. Moussa, C. et al. Resveratrol regulates neuro-inflammation and induces adaptive immunity in Alzheimer's disease. Journal of Neuroinflammation 2017 14:1 14, 1 (2017).

204. McManus, M. J., Murphy, M. P. \& Franklin, J. L. The mitochondria-targeted 
antioxidant MitoQ prevents loss of spatial memory retention and early neuropathology in a transgenic mouse model of Alzheimer's disease. Journal of Neuroscience 31, 15703-15715 (2011).

205. Ma, T. et al. Amyloid $\beta$-induced impairments in hippocampal synaptic plasticity are rescued by decreasing mitochondrial superoxide. Journal of Neuroscience 31, 5589-5595 (2011).

206. Yin, X., Manczak, M. \& Reddy, P. H. Mitochondria-targeted molecules MitoQ and SS31 reduce mutant huntingtin-induced mitochondrial toxicity and synaptic damage in Huntington's disease. Human Molecular Genetics 25, 1739-1753 (2016).

207. Snow, B. J. et al. A double-blind, placebo-controlled study to assess the mitochondria-targeted antioxidant MitoQ as a disease-modifying therapy in Parkinson\&apos;s disease. Mov Disord 25, 1670-1674 (2010).

208. Cui, M., Tang, X., Christian, W. V., Yoon, Y. \& Tieu, K. Perturbations in mitochondrial dynamics induced by human mutant PINK1 can be rescued by the mitochondrial division inhibitor mdivi-1. Journal of Biological Chemistry 285, 11740-11752 (2010).

209. Baek, S. H. et al. Inhibition of Drp1 Ameliorates Synaptic Depression, A $\beta$ Deposition, and Cognitive Impairment in an Alzheimer's Disease Model. Journal of Neuroscience 37, 5099-5110 (2017).

210. Bordt, E. A. et al. The Putative Drp1 Inhibitor mdivi-1 Is a Reversible Mitochondrial Complex I Inhibitor that Modulates Reactive Oxygen Species. Dev. Cell 40, 583-594.e6 (2017).

211. Wang, D. et al. A Small Molecule Promotes Mitochondrial Fusion in Mammalian Cells. Angew. Chem. Int. Ed. 51, 9302-9305 (2012).

212. Cartoni, R. et al. The Mammalian-Specific Protein Armcx1 Regulates Mitochondrial Transport during Axon Regeneration. Neuron 92, 1294-1307 (2016).

213. Logan, C. V. et al. Loss-of-function mutations in MICU1 cause a brain and muscle disorder linked to primary alterations in mitochondrial calcium signaling. Nature Genetics 46, 188-193 (2014).

214. Bhosale, G., Sharpe, J. A., Sundier, S. Y. \& Duchen, M. R. Calcium signaling as a mediator of cell energy demand and a trigger to cell death. Ann. N. Y. Acad. Sci. 1350, 107-116 (2015).

215. Luongo, T. S. et al. The mitochondrial $\mathrm{Na}+/ \mathrm{Ca} 2+$ exchanger is essential for Ca2+ homeostasis and viability. Nature 545, 93-97 (2017).

216. Banks, W. A. From blood-brain barrier to blood-brain interface: new opportunities for CNS drug delivery. Nat Rev Drug Discov 15, 275-292 (2016).

217. Han, X.-J. et al. CaM kinase I alpha-induced phosphorylation of Drp1 regulates mitochondrial morphology. The Journal of Cell Biology 182, 573585 (2008).

218. Coppola, T. et al. Disruption of Rab3-calmodulin interaction, but not other effector interactions, prevents Rab3 inhibition of exocytosis. The EMBO Journal 18, 5885-5891 (1999).

219. Sakaba, T. \& Neher, E. Calmodulin mediates rapid recruitment of fastreleasing synaptic vesicles at a calyx-type synapse. Neuron 32, 1119-1131 (2001).

220. Cousin, M. A. \& Robinson, P. J. Ca2+ Influx Inhibits Dynamin and Arrests Synaptic Vesicle Endocytosis at the Active Zone. J Neurosci 20, 949-957 (2000).

221. Geppert, M. et al. Synaptotagmin I: A major Ca2+ sensor for transmitter 
release at a central synapse. Cell 79, 717-727 (1994).

\section{Acknowledgements}

The authors apologise to those colleagues whose work could not be cited owing to space limitations. M.J.D. was supported by a Wellcome Trust Clinical Postdoctoral Fellowship (106713/Z/14/Z) and an Academy of Medical Sciences starter grant. This work was further supported by a grant from the Wellcome Trust (093239/Z/10/Z), an ERC starting grant (Fuelling Synapses) and a research prize from the Lister Institute of Preventive Medicine to J.T.K.

\section{Author contributions statement}

M.J.D. and J.T.K. researched data for article, discussed the content, wrote the article and reviewed and edited the manuscript before submission.

\section{Competing interests statement}

The authors declare no competing interests.

\section{Biographies}

Michael J. Devine is a Wellcome Trust Postdoctoral Clinical Fellow in Josef Kittler's laboratory, and is interested in the molecular mechanisms of neurological diseases. He completed a PhD in John Hardy's group at UCL, developing induced pluripotent stem cell models of Parkinson disease. $\mathrm{He}$ is also a consultant neurologist at the National Hospital for Neurology and Neurosurgery.

Josef Kittler is a Professor of Neurobiology in the Department of Neuroscience, Physiology and Pharmacology at UCL. His research focuses on understanding the biological cellular mechanisms that underlie how neurons communicate with each other, with the aim of improving our knowledge of how this goes wrong in neurological and psychiatric diseases.

\section{Key points}

- Synaptic communication within the nervous system is a highly energy demanding process that is tightly regulated by $\mathrm{Ca}^{2+}$ signalling.

- Mitochondria are ideally suited to provide energy to power synaptic function and buffer $\mathrm{Ca}^{2+}$ and they are actively recruited to and from synapses.

- Presynaptic mitochondria are important for providing ATP to support prolonged synaptic activity.

- They are also capable of buffering presynaptic $\mathrm{Ca}^{2+}$ signals, thereby modulating neurotransmission and potentially placing an upper limit on synaptic activity.

- Greater computational flexibility might be afforded by varying mitochondrial occupancy of presynapses.

- Dysfunction of presynaptic mitochondria could contribute to neurodegeneration by impairing synaptic homeostasis.

\section{Glossary terms}

Mitochondrial matrix: the space within the inner membrane of the mitochondrion, containing the enzymes that facilitate reactions in the citric acid cycle, oxidative phosphorylation, pyruvate oxidation and beta oxidation of fatty acids.

Readily releasable pool: the pool of synaptic vesicles that are available for immediate release, being docked at the presynaptic active zone and primed for release. 
Recyclable pool: these vesicles are recruited to the active zone, once the readily releasable pool is depleted, and maintain vesicle release under moderate stimulation.

Reserve pool: a depot of vesicles that are released only during intense stimulation, constituting the majority of vesicles in most presynaptic terminals.

Retinal bipolar neurons: cells that connect light sensitive rods or cones in the retina with ganglion cells.

Mitochondrial permeability transition pore: formed in the inner membrane of the mitochondrion under certain pathological conditions, increasing mitochondrial membrane permeability, which can lead to mitochondrial swelling and cell death.

Retinal ganglion cells: convey visual information from retinal bipolar cells to the brain.

Wallerian degeneration: the degeneration of an axon distal to the site of an injury. 


\section{Box 1 I Elimination of damaged mitochondria}

Damaged mitochondria produce excessive reactive oxygen species (ROS) that are harmful to the cell and therefore need to be eliminated. Under normal conditions, PINK1 is imported into mitochondria where it is cleaved and then digested in the proteasome. However, depolarised mitochondria fail to import PINK1, which instead accumulates on the outer mitochondrial membrane (OMM). PINK1 then

phosphorylates ubiquitin (a), which can be bound by parkin (b), facilitating parkin phosphorylation by PINK1 (c). This activates parkin's E3 ubiquitin ligase activity, enabling parkin to further ubiquitinate OMM proteins (d). This triggers autophagic machinery to engulf and digest damaged mitochondria in a process called mitophagy (reviewed in REF. ${ }^{195}$ ).

The PINK1-parkin pathway has also been implicated in mediating the pruning of oxidised cargo proteins from mitochondria in the form of mitochondria-derived vesicles (MDVs), which are targetted to lysosomes ${ }^{196}$. An apparently separate population of MDVs is targetted to peroxisomes ${ }^{197}$, although it is not clear at present why different MDVs are degraded via different pathways. Nevertheless, MDVs enable mitochondria to maintain integrity of the mitochondrial network without having to eliminate entire organelles in response to damage.

Parkin-independent mitophagy pathways have also been identified such as the recently described FKBP8-LC3A pathway ${ }^{198}$. The FUN14 domain-containing protein 1 (FUNDC1) pathway is another, activated in hypoxic conditions ${ }^{199}$ or upon exposure to acute mitochondrial toxins 200 .

Intriguingly, some cells can remove damaged mitochondria by shedding them extracellularly: mouse retinal ganglion cell axons appear to shed the majority of their damaged mitochondria at the optic nerve head, which are then internalised and degraded by neighbouring astrocytes ${ }^{201}$. This phenomenon is yet to be described in other cell subtypes, but highlights that mitophagy is not exclusively cell autonomous. 


\section{Box 2 I Therapeutic strategies targeting presynaptic mitochondria}

Pharmacological modulation of mitochondrial function is a potentially powerful way of treating a wide variety of conditions that feature mitochondrial dysfunction. General enhancement of mitochondrial biogenesis and oxidative capacity can be achieved with resveratrol, which stimulates sirtuin-1 activity (mimicking caloric restriction, which increases lifespan in a variety of organisms ${ }^{202}$ ). This has shown promise in a recent small clinical trial in Alzheimer disease (AD), attenuating decline in cognition, along with reduction in cerebrospinal fluid markers of inflammation ${ }^{203}$. MitoQ is a mitochondrially targeted form of co-enzyme Q10 (a key part of the mitochondrial electron transport chain and also an antioxidant), which has been shown to have beneficial effects in animal models of neurodegeneration, with attenuation of neuropathology and synaptic deficits in transgenic mouse models of $A D^{204,205}$ and Huntington disease ${ }^{206}$. However, so far this has not translated into demonstrable clinical improvement, at least in Parkinson disease ${ }^{207}$.

Altering the balance of mitochondrial fission-fusion might also be exploited therapeutically. For example, genetic depletion of Drp1 reduces mitochondrial dysfunction and improves synaptic function in a mouse model of $A D^{142}$. The mitochondrial division inhibitor MDIVI1, which has been widely considered to be an inhibitor of DRP1, offsets PINK1-induced mitochondrial dysfunction ${ }^{208}$ and ameliorates mitochondrial dysfunction, synaptic depression and cognitive deficits in a mouse model of $A D^{209}$. However, recently, MDIVI1 was also shown to be a complex I inhibitor (REF. ${ }^{210}$ ). Promoting fission, with $\mathrm{M} 1$ hydrazone for example ${ }^{211}$, might be a viable alternative to MDIVI1.

More specific modulation of mitochondria might prove beneficial, although this remains on the distant horizon. For example, enhancing mitochondrial trafficking in axons (via depletion of syntaphilin) increases regeneration following axonal crush injury, by replenishing healthy mitochondria where they are needed ${ }^{16}$. A further option would be to upregulate armadillo repeat-containing X-linked protein 1 (ARMCX1), which has recently been implicated in mobilising mitochondria to enhance axon regeneration following axotomy ${ }^{212}$.

Modulating precisely how mitochondria buffer $\mathrm{Ca}^{2+}$ also has great potential, and is critical to neuronal function. Loss of function of mitochondrial calcium uptake protein 1 (MICU1) leads to chronic activation of the mitochondrial calcium uniporter and causes a genetic disorder comprising muscle weakness, learning difficulties and progressive extrapyramidal motor dysfunction ${ }^{213}$. Although not yet definitively demonstrated, it is speculated that synaptic defects in individuals with this disorder might account for some of the clinical phenotype ${ }^{214}$. Other mechanisms that regulate mitochondrial $\left[\mathrm{Ca}^{2+}\right]$ also have potential as therapeutic targets: the mitochondrial $\mathrm{Na}+/ \mathrm{Ca} 2+$ exchanger is one such mechanism and recently has been shown to be essential for life 215 .

A central challenge to these strategies is the specific targeting of neuronal mitochondria. Novel methods to guide drugs to neurons across the blood-brain barrier are in development ${ }^{216}$. These will likely prove pivotal in limiting potential adverse effects of boosting mitochondrial activity in untargeted tissues, although any effects on non-neuronal cells in the brain must be kept in mind. 
Figure 1 I Schematic of a synapse. Axonal boutons and dendritic spines meet each other at synapses, to which mitochondria can be recruited via transportation along microtubules. Mitochondria provide ATP and modulate $\mathrm{Ca}^{2+}$ signals, to power and to regulate synaptic transmission, respectively. $\mathrm{Ca}^{2+}$ influx triggers fusion of synaptic vesicles in the readily releasable pool (RRP) with the plasma membrane at the active zone, emptying their contents of neurotransmitter (in this example, glutamate) into the synaptic cleft. In turn, the neurotransmitter acts on postsynaptic receptors to effect synaptic transmission. Synaptic vesicles are then endocytosed and return to the recycling pool (RP) for use on future occasions. VGCC: Voltage gated $\mathrm{Ca}^{2+}$ channel. PMCA: Plasma membrane $\mathrm{Ca}^{2+}$ ATPase. NCX: $\mathrm{Na}^{+} / \mathrm{Ca}^{2+}$ exchanger. NCLX: Mitochondrial $\mathrm{Na}^{+} / \mathrm{Ca}^{2+}$ exchanger. 
Figure 2 I Mitochondrial recruitment to the presynapse. a I Mitochondrial transport along microtubules is mediated by mitochondrial Rho GTPases (MIROs), which directly bind to kinesin and trafficking kinesin-binding proteins (TRAKs) and indirectly interact with dynein via TRAKs and $\mathrm{p} 150$. The TRAKs also bind $\mathrm{N}$ acetylglucosamine transferase (OGT). The exact mechanism of $\mathrm{Ca}^{2+}$-dependent mitochondrial arrest is unknown but three models have been suggested. $\mathbf{b}$ I In one model, MIRO1 binds directly to kinesin heavy chain isoform 5 (KIF5), but this is inhibited by rising $\mathrm{Ca}^{2+}$ levels, thus uncoupling mitochondria from the motor transport pathway at sites of increased $\mathrm{Ca}^{2+}\left(\mathrm{REF}^{22}\right)$. c I In a second model, MIRO interacts with KIF5 via TRAKs and increased $\mathrm{Ca}^{2+}$ causes KIF5 to release from microtubules and instead interact with mitochondrial $\mathrm{MIRO}^{23}$. d I MIRO binding of $\mathrm{Ca}^{2+}$ leads to a dissociation from KIF5 motors, which can then in turn interact with the axonal tethering protein syntaphilin, anchoring mitochondria to the microtubule track ${ }^{12}$. 
Figure 3 I Generation and consumption of ATP in presynaptic terminals. The majority of presynaptic ATP is generated by mitochondria via oxidative phosphorylation (OXPHOS), which is a more efficient means of converting glucose to usable energy ( $\sim 30$ molecules of ATP formed per glucose molecule) in comparison to glycolysis (2 molecules of ATP per molecule glucose). This currency of cellular energy is then used to power the activities of the presynapse, including mitochondrial fission and fusion, vesicular exocytosis, endocytosis and recycling, and the reversal of ion fluxes through voltage-gated and ligand-gated ion channels that subserve synaptic transmission. The loss or dysfunction of presynaptic mitochondria causes local energy deficits, thus impairing presynaptic function. 
Figure 4 I Regulation of $\mathbf{C a}^{2+}$ transients at the presynapse. $\mathrm{Ca}^{2+}$ is tightly regulated by interacting mechanisms for $\mathrm{Ca}^{2+}$ transport located in mitochondria, endoplasmic reticulum (ER) and the plasma membrane. Vesicle-associated membrane-associated protein B (VAPB)-protein tyrosine phosphatase-interacting protein 51 (PTPIP51) and inositol trisphosphate (IP3)-voltage-dependent anionselective channel (VDAC) interactions (the latter facilitated by GRP75) in mitochondrial associated ER membrane (MAM) permit transfer of $\mathrm{Ca}^{2+}$ from the ER to mitochondria ${ }^{104}$. The mitochondrial calcium uniporter (MCU) in the inner mitochondrial membrane (IMM) enables mitochondrial $\mathrm{Ca}^{2+}$ uptake from the cytosol. ER can also take up $\mathrm{Ca}^{2+}$ via sarco/endoplasmic reticulum $\mathrm{Ca}^{2+}$ ATPase (SERCA) pumps. Similarly, $\mathrm{Ca}^{2+}$ can be extruded from the cytosol via plasma membrane $\mathrm{Ca}^{2+}$ ATPase (PMCA). The $\mathrm{Na}^{+} / \mathrm{Ca}^{2+}$ exchange (NCX) pump utilises the $\left[\mathrm{Na}^{+}\right]$gradient across the plasma membrane to augment cytosolic $\mathrm{Ca}^{2+}$ extrusion. The mitochondrial $\mathrm{Na}^{+} / \mathrm{Ca}^{2+}$ exchanger (NCLX) plays a key role in mitochondrial $\mathrm{Ca}^{2+}$ extrusion. The ER can also influence plasma membrane $\mathrm{Ca}^{2+}$ regulation by sensing $\mathrm{Ca}^{2+}$ depletion via STIM1 proteins, which then translocate to ER-plasma membrane junctions and recruit $\mathrm{Ca}^{2+}$-release activated $\mathrm{Ca}^{2+}(\mathrm{CRAC})$ channels permitting $\mathrm{Ca}^{2+}$ influx. In turn, $\mathrm{Ca}^{2+}$ impacts on a plethora of downstream effector proteins to regulate presynaptic activity: CaMK1a phosphorylates DRP1 to upregulate mitochondrial fission ${ }^{217}$, calmodulin interacts with RAB GTPases to modulate vesicle recycling and replenishment of the $R_{R P} 218,219$, dynamin inhibits vesicle endocytosis ${ }^{220}$, and synaptotagmin promotes vesicular release 221 . These, at times, opposing functions highlight the importance of tight spatial, as well as temporal, regulation of $\mathrm{Ca}^{2+}$. 
Figure 5 I Pathogenesis of disease. a I Alzheimer disease (AD) features aggregation of the peptide amyloid $\beta(A \beta)$ and extracellular deposition of amyloid plaques. Arrows depict the cellular processes that $A \beta$ can affect. $A \beta$ oligomers can influence the mitochondrial-associated endoplasmic reticulum (ER) membrane (MAM), increasing mitochondria-ER interactions and augmenting ER to mitochondrial $\mathrm{Ca}^{2+}$ transfer ${ }^{157}$. AD brain features upregulated insulin-like growth factor 1 receptor (IGF1R) signalling, which increases mitochondrial $\mathrm{Ca}^{2+}$ uptake and ATP release, impacting synaptic transmission ${ }^{93}$. Mitochondrial fission is also affected: oligomeric $A \beta$ upregulates dynamin-related protein 1 (DRP1) via nitric oxide (NO) signalling ${ }^{140}$, whereas downstream tau pathology attenuates dynamin-related protein 1 (DRP1) activity by increasing its propensity to remain actin bound ${ }^{143}$.

Phosphorylated tau also inhibits anterograde mitochondrial movement ${ }^{145}$. b I

Parkinson disease (PD) features misfolding and aggregation of a-synuclein, a protein that is localised to MAMs (binding to VAPB) but relocates under pathological conditions, impairing MAM function ${ }^{183}$. a-Synuclein overexpression inhibits complex I and depolarises mitochondria leading to their fragmentation ${ }^{177}$. a-Synuclein overexpression inhibits synaptic vesicle release ${ }^{181}$. PINK1 deficiency can also impair neurotransmitter release by inhibiting complex I, thereby reducing mitochondrial energy production ${ }^{173}$. Dysfunctional mitochondrial quality control in PD is highlighted by disease-causing mutations in PINK $1^{159}$ and $P A R K 2^{160}$, which encodes parkin, both of which are required for appropriate tagging of mitochondria for mitophagy. 
 \\ Figure 1: Schematic of a synapse}
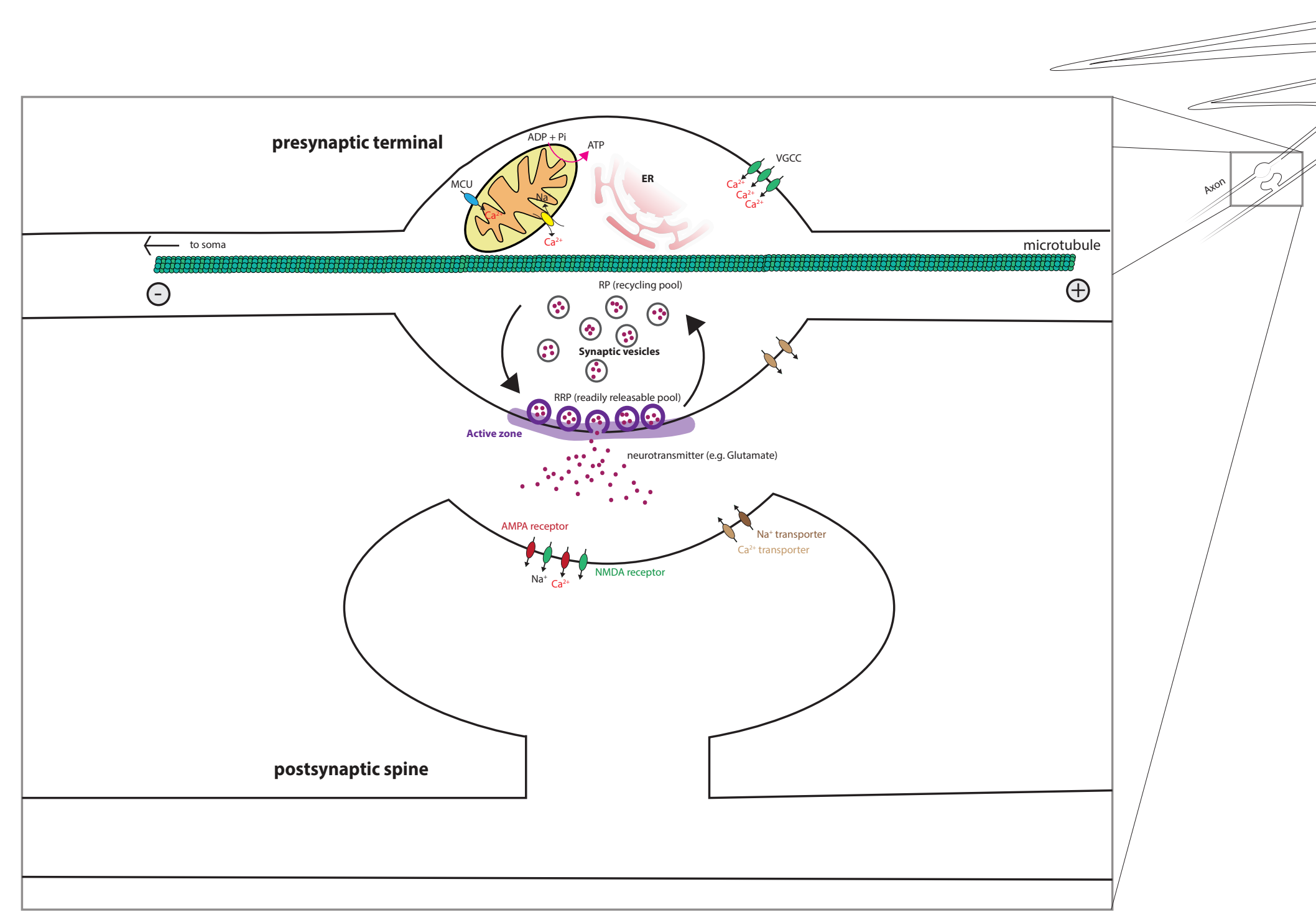
Figure 2: Mitochondrial trafficking machinery

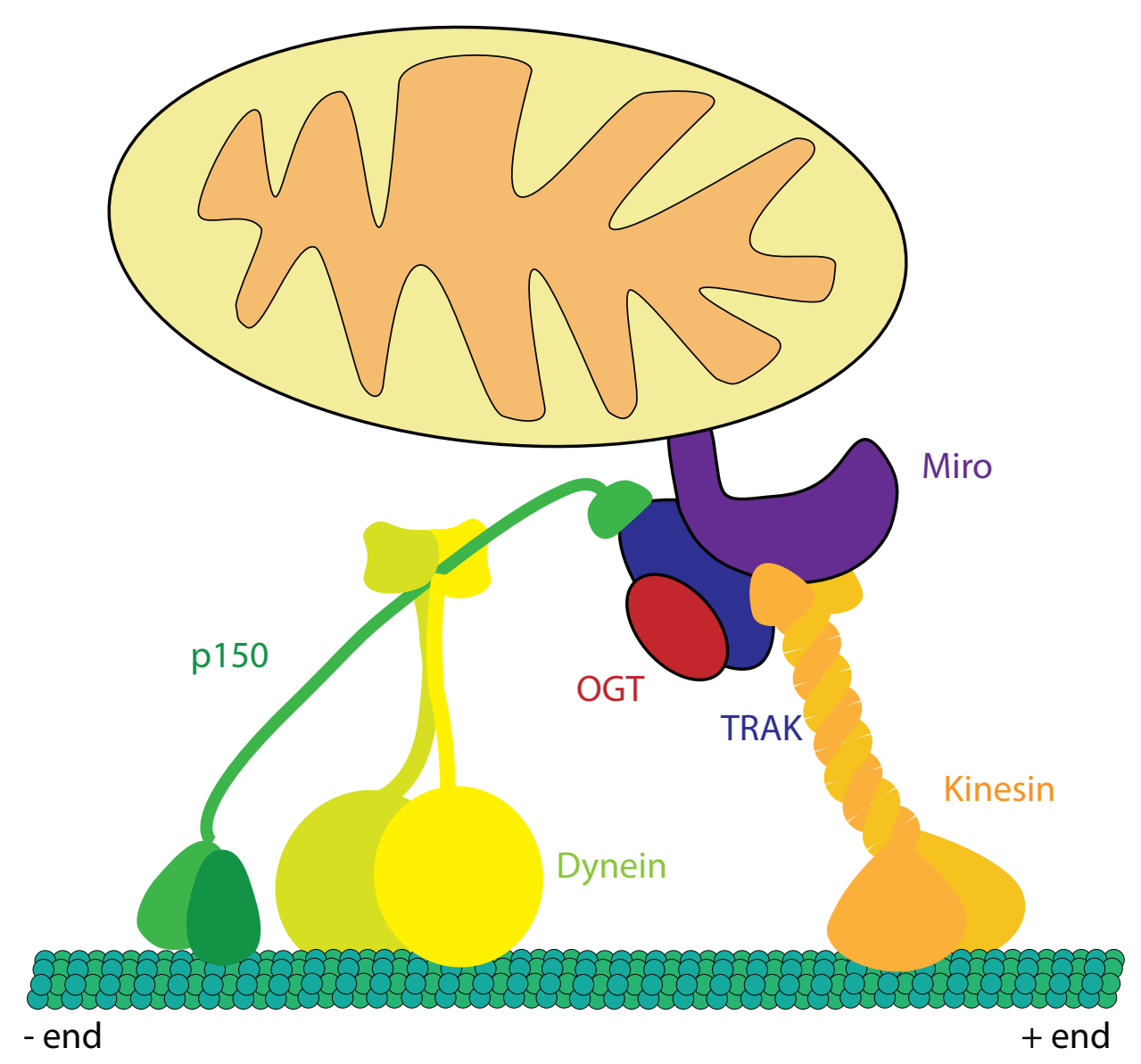

a
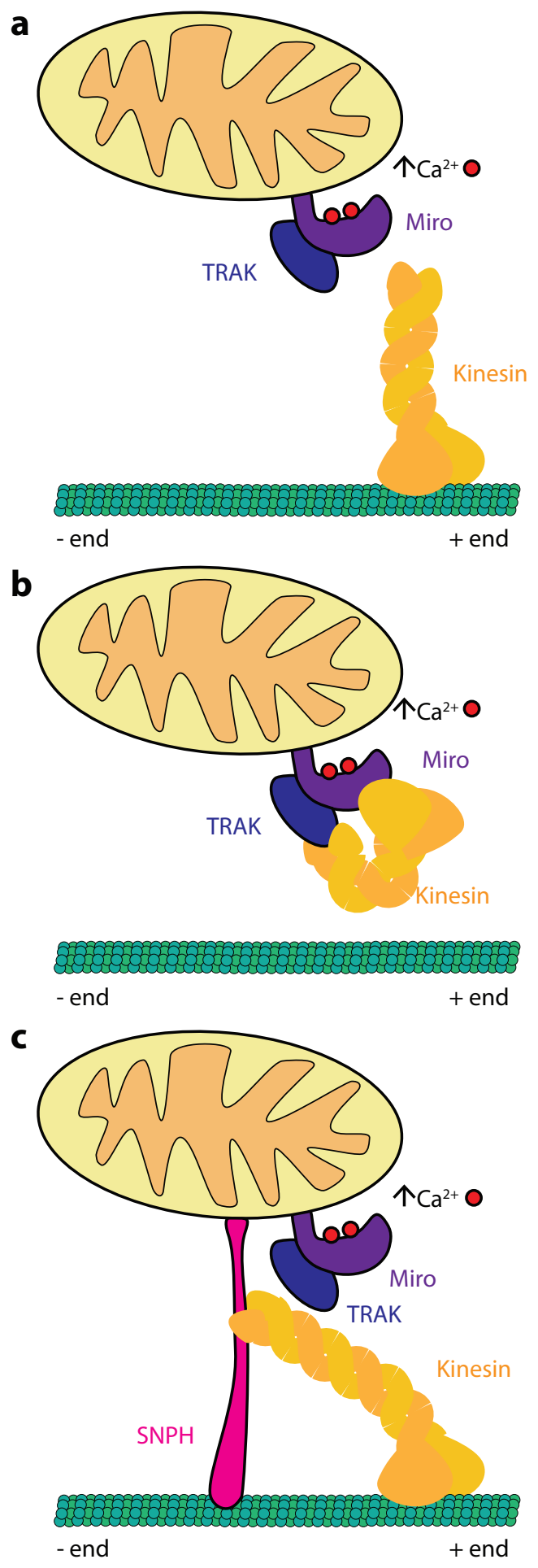
Figure 3: Schematic of a syndpse

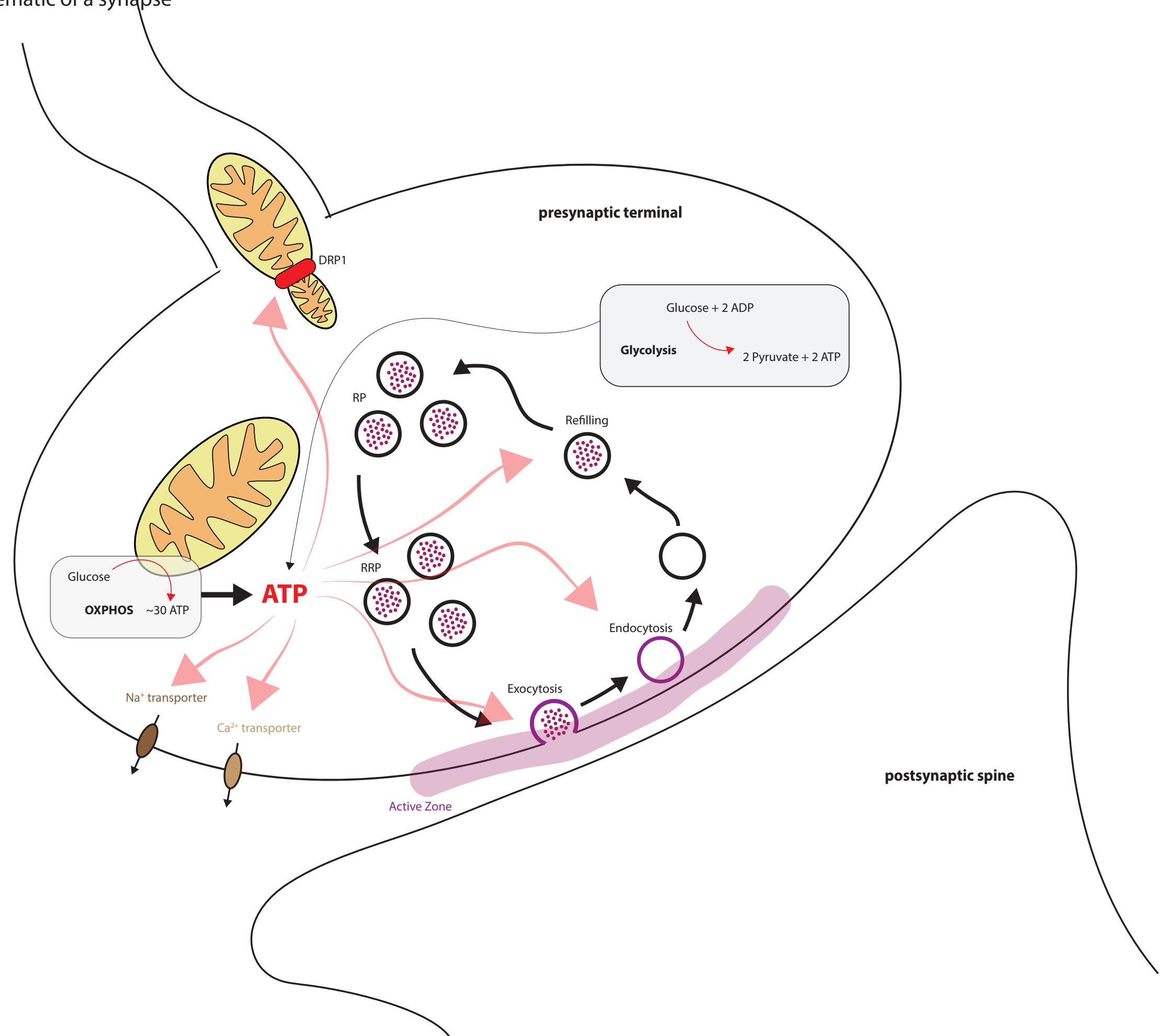



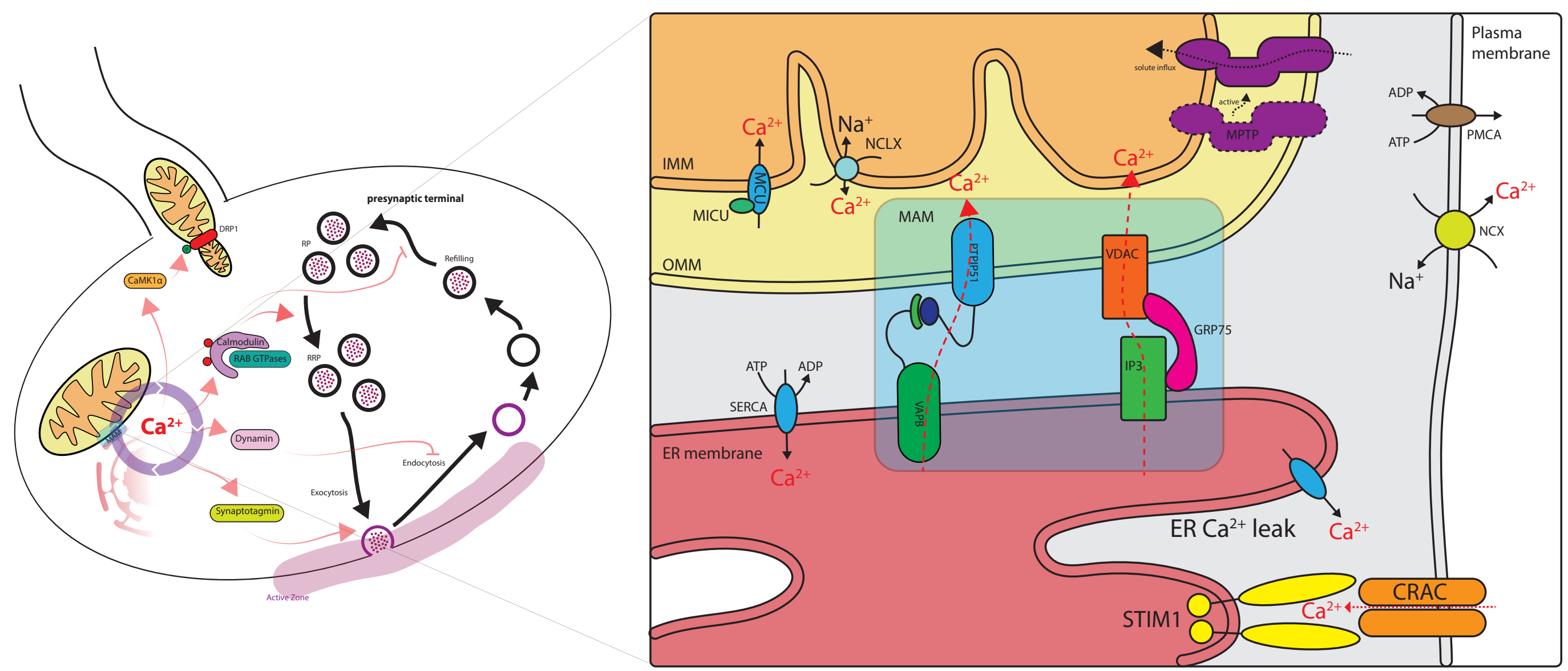

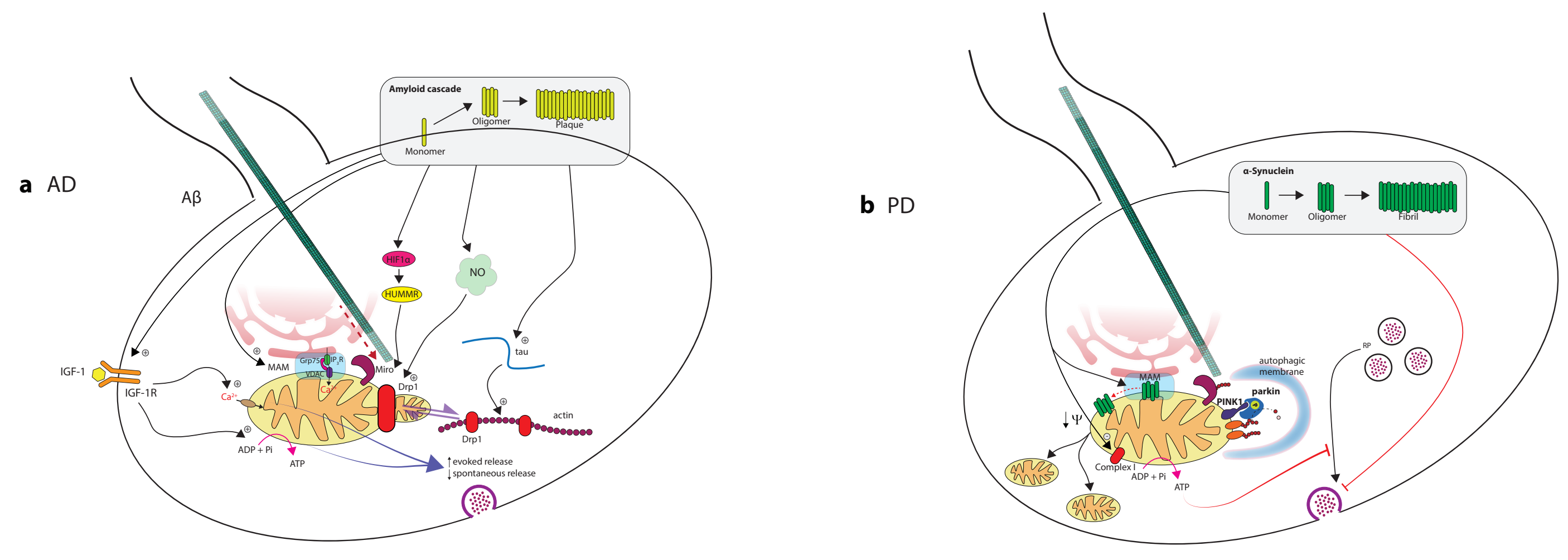